\title{
Statistical variability of mechanical fields in thermo-poro-elasticity: multiscale analytical estimations applied to cement-based materials at early-age
}

\author{
Tulio Honorio ${ }^{\mathrm{a}, *}$, Laurent Brochard ${ }^{\mathrm{a}}$, Benoît Bary ${ }^{\mathrm{b}}$ \\ ${ }^{a}$ Université Paris-Est, Laboratoire Navier (UMR 8205), CNRS, ENPC, IFSTTAR, $6 \& 8$ \\ Avenue Blaise Pascal, 77455 Marne-la-Vallée, France \\ ${ }^{b}$ Den-Service dEtude du Comportement des Radionucléides (SECR), CEA, Université \\ Paris-Saclay, F-91191, Gif-sur-Yvette, France
}

\begin{abstract}
Analytical homogenization provides information about the average mechanical fields but can also be used to obtain information about the statistical variability or fluctuations of these fields. Here, we present analytical estimations of the fluctuations of elastic stresses within cement-based materials (CBM). MoriTanaka, self-consistent and generalized self-consistent schemes are combined to represent the multiscale character of CBM. The second moment of mechanical fields is derived from the internal energy. The presence of transformation fields and resulting fluctuations are considered. We account for the evolution of the volume fractions of phases due to cement hydration. Scenarios considering or not the ITZ are studied. Our estimations are dependent on load and representation CBM microstructure. The obtained standard deviation of stresses in C-S-H are on the order of the stresses applied at concrete scale. We provide estimations of the compressive strength of a cement paste at early-age using our results of fluctuation of stresses.
\end{abstract}

Keywords: field fluctuations; thermo-poro-elasticity; early-age;

*Corresponding author; Current address: Université Paris-Est Créteil, Laboratoire Modélisation et Simulation Multi-Echelle, MSME UMR 8208 CNRS, 94010, Créteil cedex, France; tulio.honorio-de-faria@u-pec.fr

Email address: tuliohfaria@gmail.com; tulio.honorio-de-faria@enpc.fr (Tulio Honorio)

Preprint submitted to Cement and Concrete Research

March 14, 2018 
homogenization; compressive strength.

\section{Highlights}

- Fluctuations of mechanical fields are estimated by analytical homogenization

- Standard deviation of stresses in C-S-H has the order of magnitude of the stresses applied at concrete scale.

- Fluctuations increase with the stiffness and contrast of phases.

- Fluctuations are dependent of mechanical load.

- Transformation fields also cause mechanical fluctuations.

\section{Introduction}

The heterogeneous and hierarchical multi-scale character of Cement-Based Materials (CBM) is closely related to key properties affecting thermo-mechanical performance of such materials. Upscaling techniques have been applied to the estimations of effective properties of CBM including elastic [1, 2, 3, 4, ageing and non-ageing creep and relaxation functions [5, 6, 7, 8, diffusion [9] and thermal properties (thermal conductivity and heat capacity) [10, 11] as well as coupling properties such as Biot coefficient and coefficients of thermal expansion [10, 12. These estimations focus on first-order or averaged information per phase, without coping with the distribution of fields (notably, mechanical fields) within phases.

The distribution of fields within a phase (intraphase distribution) is, though, a useful information concerning the development of more precise multiscale estimations. This local information is valuable in the study of composite materials in which some constituents may have stress or strain states exceeding some criterion determining the onset of damage or failure. Indeed, fluctuations in 
mechanical fields are reported to play a major role in the determination of failure mechanisms [13]. The computation of second order moments of mechanical fields (from which fluctuations are obtained) is proven to be a crucial ingredient in the development of more precise constitutive models of porous materials [14. Upscaling estimations of CBM strength require the computations of these so second moments [15, 16, 17, 18]. However, in such studies no detailed analysis of the factors affecting the statistical variability of mechanical fields per se is provided. Information about the statistical variability of mechanical fields can be used to test important hypothesis regarding the mechanical behaviour of CBM. For instance, the heterogeneity of mechanical fields is associated with key aspects of CBM behavior such as viscoelastic deformations (through the relaxation of prestresses [19, 20, 21] or microfissuration [22], for instance) and eventual non-linearities of the mechanical behavior. Moreover, the prediction of the heterogeneity of mechanical fields can be useful in understanding durability issues, especially those associated with swelling reactions such as alkali-silica 40 reaction.

Numerical simulations have been employed to get local information within CBM. For example, in [6], finite elements methods is used to compute the averaged stresses and strains in sub-volumes of a numerical sample representing concrete - a distribution of the mechanical fields within aggregates and matrix 45 (mortar) is then obtained. But as for ad-hoc modelling, the results of numerical simulations may lack of generality.

Analytical homogenization tools may also provide information about the fluctuations (from which a standard deviation can be estimated) of mechanical fields within a given phase. General estimations in the presence or not of transformation fields have been proposed in the litterature $[23,24,25,26]$. However, to the authors knowledge, no analytical estimations of the fluctuations of mechanical fields applied to CBM were proposed to date. Note that these theoretical estimations have been used to validate numerical estimations (e.g. in the Finite Elements code developed by Garboczi [27]), since in some situations such ${ }_{55}$ as biphasic composites, theoretical results are exact. Furthermore, mechanical 
fields measurements in multiscale context, specially stress fields and residual stresses are recognized for being difficult to measure directly (e.g. 28]). Modelling, including theoretical modelling arises as a powerful tool to fill this gap. Indeed, most of the developments of stress fluctuations theory [23, 24, 25, 26] have been made in a purely theoretical framework and could provide important insight into the behaviour of various materials.

In this paper, we estimate inter- and intra-phase distributions of strain and stresses fields following a multiscale representation of CBM in elasticity and in the presence of transformation fields. We provide a detailed analysis of the factors affecting the statistical variability of mechanical fields in CBM (namely, property contrast, age, phase assembly, morphology) and its estimates (e.g. choice of homogenization scheme). CBM have a multi-scale porosity with pore sizes ranging from few Angstroms (in C-S-H interlayer pores) up to few millimeters (in air trapped voids) which justifies the application of poromechanics to the study of such materials [29]. Also, structures composed of CBM are subject to thermal loadings in their service lives due to different transfert phenomena [30]. Therefore, we consider problems in thermoelasticity and poroelasticity. The general formulation of these problems is first presented. The localization relations of the analytical schemes employed are recalled. Then, the formula75 tion for the estimation of the variance of the mechanical fields, following [25], is presented. We assume micro- and macro-isotropy; and the estimations of fluctuations are made for spherical or equiaxed particles. We first compare our results to simulations performed on numerical samples to obtain local information on the distribution of mechanical fields. The representation of the multi-scale character of CBM adopted and the tools used to model hydration evolution is based on previous work and is briefly recalled [6, 10, 31]. Applications to cement paste, ITZ, mortar and concrete scales are finally held out. The applications include estimations of compressive strength of cement paste and their confrontation with experimental data from the literature. 


\section{Overall and local mechanical behaviour in the presence of trans- formation fields}

Consider a composite with elastic constituent phases, which are perfectly bonded at their interfaces. We assume that the properties of these phases are statistically independent quantities. Let such composite be homogeneous at a given macro-scale, so that a Representative Element Volume (REV) $\Omega$ can be chosen to study local and overall behaviour. In terms of Cauchy stress and linearized (infinitesimal) strain, the overall behaviour is:

$$
\sigma(\mathbf{x})=\mathbb{C}(\mathbf{x}): \varepsilon(\mathbf{x})+\sigma^{(0)}(\mathbf{x}), \forall x \in \Omega
$$

or, equivalently

$$
\varepsilon(\mathbf{x})=\mathbb{S}(\mathbf{x}): \sigma(\mathbf{x})+\varepsilon^{(0)}(\mathbf{x}), \forall x \in \Omega
$$

where $\mathbb{C}$ and $\mathbb{S}$ are, respectively, the symmetric fourth-rank elastic stiffness and compliance tensors, which are the reciprocal of each other $\left(\mathbb{C}=\mathbb{S}^{-1}\right) ; \sigma^{(0)}$ and $\varepsilon^{(0)}$ are the overall stress and strain transformation fields (or eigenstress and eigenstrain), respectively. At this stage, no specific assumptions regarding the geometry of the microstructure are adopted. The tranformation fields in the affine form of Eqs. 1 and 2 may represent different physical phenomena including deformations due to a uniform change in temperature, phase transformation or other changes in the volume or shape of the material [32, 26]. Here, we are particularly interested in prestresses or prestrains due to thermoelasticity and (saturated) poroelasticity, for which the transformation stresses and strains are written, respectively, as:

$$
\begin{gathered}
\sigma^{(0)}(\mathbf{x})=-\mathbb{C}(\mathbf{x}): \mathcal{A}(\mathbf{x})\left(T-T_{0}\right) ; \varepsilon^{(0)}(\mathbf{x})=\mathcal{A}(\mathbf{x})\left(T-T_{0}\right) \\
\sigma^{(0)}(\mathbf{x})=-B(\mathbf{x}) p ; \varepsilon^{(0)}(\mathbf{x})=\mathbb{S}: B(\mathbf{x}) p
\end{gathered}
$$


Concerning thermoelastic behaviour, $\mathcal{A}$ is the tensor of the coefficients of thermal expansion; $T$ is the current temperature and $T_{0}$ is a temperature of reference. Concerning poroelastic behaviour, $B$ is the tensor of Biot coefficients; $p$ is the pressure in the fluid filling the pores.

\subsection{Local behaviour and residual stresses}

The local constitutive relation, in a sub-volume of $\Omega_{s} \in \Omega$, reads:

$$
\sigma(\mathbf{x})=\mathbb{B}(\mathbf{x}): \Sigma+\sigma^{r e s}(\mathbf{x}), \forall x \in \Omega_{s}
$$

where $\mathbb{B}$ is the stress localization forth-rank tensor; $\Sigma$ is a uniform stress applied at the boundary $\partial \Omega$ of the RVE and $\sigma^{r e s}(\mathbf{x})$ is the residual stress field resulting from the incompatibility of the a local eigenstrain $\varepsilon_{s}^{(\mathbf{0})}$. As pointed out by [32], with the assumption of a uniform load applied on $\partial \Omega$, the local transformation and residual fields that are admissible in $\Omega$ must generate a uniform overall strain at the macroscopic scale. Adopting a "mean-field" approach, by considering the average fields within a given phase $(i)$, one might write

$$
\langle\sigma\rangle_{i}=\langle\mathbb{B}\rangle_{i}: \Sigma+\left\langle\sigma^{r e s}\right\rangle_{i}
$$

where $\langle.\rangle_{x}=\int_{x} . d x$ stands for the volume average over a volume $x$. With this equation the interphase distribution of the stress within a composite is obtained. In order to estimate both volume averages in the second term of last equation, we can resort to the well-known Eshelby solution for an ellipsoidal inclusion in an infinite homogeneous linear medium under a homogeneous loading $\Sigma$ [33]. Thus, without specifying a homogenization scheme, the phase averaged stress localization tensor reads (e.g. 34]):

$$
\langle\mathbb{B}\rangle_{i}=\left(\mathbb{S}_{i}+\mathbb{H}_{\mathrm{R}}\right)^{-1}:\left\langle\left(\mathbb{S}_{i}+\mathbb{H}_{\mathrm{R}}\right)^{-1}\right\rangle_{\Omega}^{-1}
$$

${ }_{125}$ where $\mathbb{H}_{\mathrm{R}}$ is the inverse of Hill's 35] constraint tensor $\mathbb{H}_{\mathrm{R}}=\left(\left(\mathbb{S}_{\mathrm{R}}^{\mathrm{E}}\right)^{-1}-\mathbb{I}\right)^{-1}: \mathbb{S}^{*}$, with $\mathbb{S}_{\mathrm{R}}^{\mathrm{E}}$ being the Eshelby tensor, $\mathbb{S}^{*}$ being the compliance of the reference 
medium and $\mathbb{I}$ being the identity forth-rank tensor. $\mathbb{S}^{*}$ is defined in line with the chosen homogenization scheme.

According to a given reference medium, the effective compliance can de determined by:

$$
\mathbb{S}^{\mathrm{eff}}=\langle\mathbb{S}: \mathbb{B}\rangle_{\Omega}=\sum_{i=1}^{N} f_{i} \mathbb{S}_{i}:\langle\mathbb{B}\rangle_{i}
$$

where the sum term can be written under the assumption of piecewise uniform fields inside a phase.

Finally, it can be shown [36, 37] that the average residual stress tensor may be obtained from the effective properties of the medium and the averaged stress localization tensors $\langle\mathbb{B}\rangle_{i}$ :

$$
\left\langle\sigma^{r e s}\right\rangle_{i}=\left(\mathbb{S}_{i}+\mathbb{H}_{\mathrm{R}}\right)^{-1}:\left(\left\langle{ }^{t}\langle\mathbb{B}\rangle_{i}: \varepsilon_{i}^{(0)}\right\rangle_{\Omega}-\left\langle\varepsilon_{i}^{(0)}\right\rangle\right)
$$

with the superscript " $t$ "standing for the transpose of a tensor.

\subsection{Isotropic medium and homogenization schemes}

For an isotropic medium the stiffness (or its reciprocal, the compliance) can be decomposed in hydrostatic and deviatoric parts:

$$
\mathbb{C}_{i}=3 k_{i} \mathbb{J}+2 \mu_{i} \mathbb{K}
$$

where $k$ and $\mu$ are the bulk and shear modulus, respectively; $\mathbb{J}$ and $\mathbb{K}$ are the forth-rank tensor extracting the volumetric and deviatoric part of a second rank tensor.

Assuming that the behaviour of the constituent phases is isotropic and that the phases are randomly distributed over the REV, the macroscopic behaviour is also isotropic. In the following we recall classical estimations under the hypothesis of micro- and macro-isotropy. 


\subsubsection{Schemes based on Eshelby solution: Mori-Tanaka and Self-Consistent} schemes for spherical inclusions

Let $\mathbb{S}^{*}$ be the stiffness tensor of the infinite medium. With the Self-Consistent $(\mathrm{SC})$ estimate, the reference medium is the effective medium $\mathbb{S}^{*}=\overline{\mathbb{S}}$. With Mori-Tanaka (MT) estimate the matrix is the reference medium $\mathbb{S}^{*}=\mathbb{S}^{0}$. For both schemes, which are based on Eshelby solution, the effective bulk and shear modulus are, respectively:

$$
k^{E S}=\sum_{i}^{N} f_{i} k_{i} A_{i}^{k} \quad \mu^{E S}=\sum_{i}^{N} f_{i} \mu_{i} A_{i}^{\mu}
$$

where $A_{i}^{k}$ and $A_{i}^{\mu}$ are the hydrostatic and deviatoric components, respectively, of the strain localization tensor given by:

$$
\begin{aligned}
A_{i}^{k} & =\frac{\left(1+a_{0}^{k}\left(k_{i} / k_{(0)}-1\right)\right)^{-1}}{\sum_{i}^{N} f_{r}\left(1+a_{0}^{k}\left(k_{i} / k_{(0)}-1\right)\right)^{-1}} \\
A_{i}^{\mu} & =\frac{\left(1+b_{0}^{\mu}\left(\mu_{i} / \mu_{(0)}-1\right)\right)^{-1}}{\sum_{i}^{N} f_{r}\left(1+b_{0}^{\mu}\left(\mu_{i} / \mu_{(0)}-1\right)\right)^{-1}}
\end{aligned}
$$

with

$$
a_{0}^{k}=\frac{3 k_{(0)}}{3 k_{(0)}+4 \mu_{(0)}} \quad b_{0}^{\mu}=\frac{6\left(3 k_{(0)}+2 \mu_{(0)}\right)}{5\left(3 k_{(0)}+4 \mu_{(0)}\right)}
$$

where $k_{(0)}$ and $\mu_{(0)}$ the bulk and shear modulus of the reference medium. For the MT scheme, the reference medium is the matrix; for SC scheme, the reference medium is the homogenized medium itself, therefore the formulation is not explicit.

The isotropic effective Biot's coefficient $b$ can be estimated by the simplified relation :

$$
b=1-\left(\sum_{i}^{N} f_{i} A_{i}^{k}\right)
$$

The isotropic effective coefficient of thermal expansion $\alpha$ is given by [38, 39]:

$$
\alpha^{E S}=\sum_{i}^{N} f_{i} \alpha_{i} B_{i}^{k}
$$


where $B_{i}^{k}$ is the hydrostatic component of the stress localization tensor given by:

$$
B_{i}^{k}=\frac{k_{i}\left(1+a_{0}^{k}\left(k_{i} / k_{(0)}-1\right)\right)^{-1}}{\sum_{i}^{N} f_{i} k_{i}\left(1+a_{0}^{k}\left(k_{i} / k_{(0)}-1\right)\right)^{-1}}
$$

\subsubsection{Generalized Self-Consistent scheme}

Solutions of effective properties of a composite sphere with $n$-concentric coats (i.e. $(n+1)$-composite accounting for the spherical core), the so-called Generalized Self-Consistent (GSC) scheme, are proposed by [40]. The bulk modulus 170 is derived by iteratively applying the formula [41]:

$$
k^{n-G S C}=k_{n-1} \frac{f_{n}^{i n}}{\frac{1}{k_{n}-k_{n-1}}+\frac{3\left(1-f_{n}^{i n}\right)}{3 k_{n-1}+3 \mu_{n-1}}}
$$

where $f_{n}^{i n}$ is the equivalent volume fraction of phase $\mathrm{n}$ with respect to the inner composite sphere. The expressions for the shear modulus are more complex and should be determined according to the number of coatings. For a 2-coated sphere, the effective shear modulus can be obtained from a quadratic equation 175 as in [42]. Once the average isotropic localization tensors [40, 38] are obtained for this scheme the formula in Eq. 15 and 16 can also be applied.

\section{Fluctuations of fields: estimations of intraphase field distributions}

Besides averaged information per phase (or interphase distribution of fields, as in Eq. 6 for stress field), more precise local information regarding the inanalytical methods. Let the fluctuation or covariance (forth-rank) tensor of a stress field within phase $(i)$ be defined by

$$
q_{i} \equiv\langle\sigma \otimes \sigma\rangle_{i}-\langle\sigma\rangle_{i} \otimes\langle\sigma\rangle_{i}
$$

where " $\otimes "$ denotes the dyadic product. Note that, even if the constituents are isotropic, the fluctuation field is anisotropic [24]. The fluctuations are larger in 

direction. The standard deviation $\omega_{i}$, or the statistical variability, of a field in a given direction $k l m n$ can be approximated by the square root of the components of the fluctuation tensor defined in Eq. 19 [25]:

$$
\omega_{i, k l m n} \equiv\left(q_{i, k l m n}\right)^{1 / 2}
$$

As shown in the last sections, the average stress $\langle\sigma\rangle_{i}$ per phase can be obtained through localization relations. In order to compute the second moment (i.e. $\langle\sigma \otimes \sigma\rangle_{i}$ ), we use the definitions of local $u$ and effective $U$ elastic energies 43]:

$$
\begin{gathered}
u(\mathbf{x})=\frac{1}{2}\left[\sigma(\mathbf{x}):\left(\varepsilon(\mathbf{x})-\varepsilon^{(0)}(\mathbf{x})\right)\right]=\frac{1}{2}[\sigma(\mathbf{x}) \otimes \sigma(\mathbf{x})]:: \mathbb{S}(\mathbf{x}) \\
U=\langle u(\mathbf{x})\rangle=\frac{1}{2}\left[\Sigma: \mathbb{S}^{\text {eff }}: \Sigma-\left\langle\sigma^{\text {res }}(\mathbf{x}): \varepsilon^{(0)}\right\rangle\right]
\end{gathered}
$$

where the effective elastic energy is obtained from the local energy by applying Hill's lemma 43 .

The second moment of stress field in a phase $i$ can be then obtained by deriving $U$ with respect to the local compliance $\mathbb{S}_{i}[26$ :

$$
\langle\sigma \otimes \sigma\rangle_{i}=-\frac{2}{f_{i}} \frac{\partial U}{\partial \mathbb{S}_{i}}
$$

Assuming $\varepsilon^{(0)}$ constant per phase, the second moment of stresses is [26, 44, 43]:

$$
\langle\sigma \otimes \sigma\rangle_{i}=\frac{1}{f_{i}}\left[\Sigma \otimes \Sigma:: \frac{\partial \mathbb{S}^{\text {eff }}}{\partial \mathbb{S}_{i}}+\sum_{i=1}^{N} f_{i} \varepsilon_{i}^{(0)}:\left(2 \Sigma: \frac{\partial^{t}\langle\mathbb{B}\rangle_{i}}{\partial \mathbb{S}_{i}}+\frac{\partial\left\langle\sigma_{\text {res }}\right\rangle_{i}}{\partial \mathbb{S}_{i}}\right)\right]
$$

This results can be used with different homogenization schemes.

The influence of the shape of inclusion on the fluctuations of stresses in the absence of transformation fields can be evaluated by considering the derivative $\frac{\partial \mathbb{S}^{\text {eff }}}{\partial \mathbb{S}_{i}}$. Strategies to solve this derivative are provided in [45, 44]. Within a 
spheroidal particles with orthogonal dimension $a_{1}=a_{2}$ and $a_{3}$, for concentrations in which the interaction between particles play a crucial role (i.e. larger volume fractions of inclusions), the average stresses in the direction of smallest inclusion size are larger than their respective values in spherical inclusions; while the average stresses in the direction of largest inclusion size are smaller than their respective values in spherical inclusions. For small volume fractions, this effect on average stresses may be inverted (see Fig. 3 and 4 in 45). The stress fluctuations are, in all cases, larger in the direction of smallest inclusion size than in the other directions. In these other direction, these fluctuations can be non-monotonic. The distribution of stresses within an ellipsoidal inclusion, accounting for the contributions of all the directions, is expected to be broader than within a spherical inclusion. Figure 1 displays a schematic representation of this effect.

The second moment of strain fields can also be obtained considering a uniform strain field on $\partial \Omega$ [43] or by combining Eq. 21 with the last equation under the assumption of macro-homogeneity [4] :

$$
\langle\varepsilon \otimes \varepsilon\rangle_{i}=\mathbb{S}_{i}:\langle\sigma \otimes \sigma\rangle_{i}: \mathbb{S}_{i}+\mathbb{S}_{i}:\langle\sigma\rangle_{i} \otimes \varepsilon_{i}^{(0)}+\varepsilon_{i}^{(0)} \otimes \mathbb{S}_{i}:\langle\sigma\rangle_{i}+\varepsilon_{i}^{(0)} \otimes \varepsilon_{i}^{(0)}
$$

From both previous equations, it can be seen that in the absence of external mechanical loading $\Sigma$, fluctuations within the phase exist if the residual stresses are non-zero. These results regarding second moments are valid to any degree of anisotropy [26]. In the macro-isotropic case, for spherical inclusions, the expressions are reduced [26] to the ones presented by [24] in the absence of transformation fields or [25] in the presence of transformation/residual fields.

The expressions regarding the latter case are shown in Appendix A and used hereafter to the computation of the fluctuations.

\subsection{Fluctuations in poromechanics}

Equations 24 and 25 deserve a closer analysis in the context of poromechanics. With Eq. 24, in the absence of external loading, fluctuations can exist due 

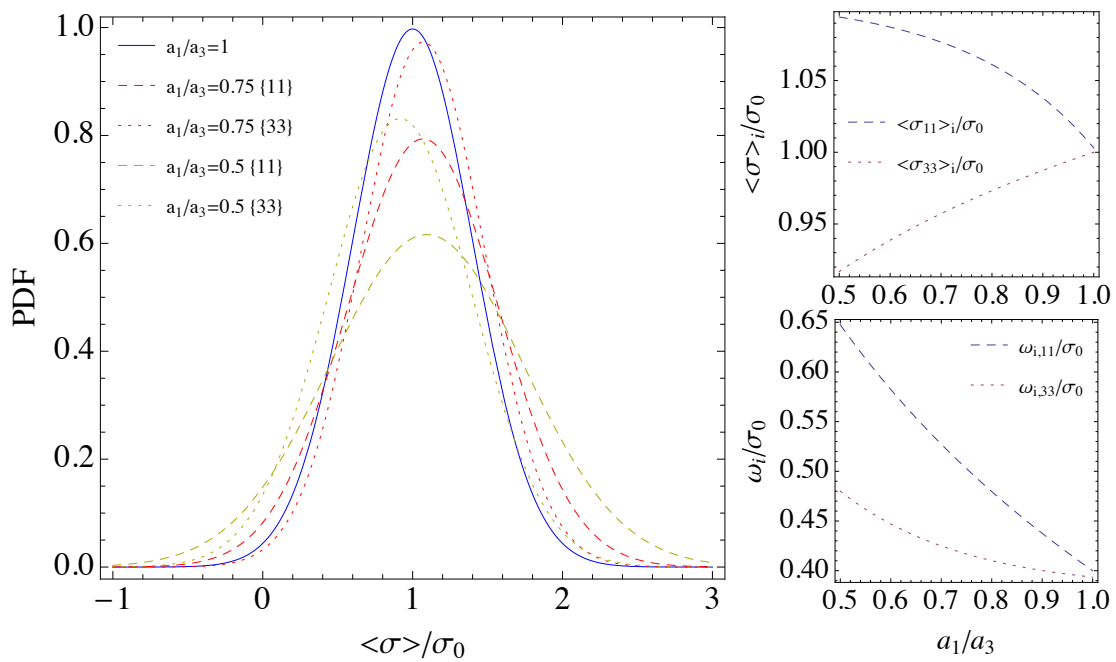

Figure 1: Sketch of the distribution of stresses in spheroidal inclusions inspired by the results of Buryachenko and Kreher 45 for concentrations in which the binary interaction between particles play a crucial role. The orthogonal dimensions of the inclusion are $a_{1}=a_{2}$ and $a_{3}$. The distribution of stresses are computed in each direction of the particle $11=22$ and 33 . 

shear modulus) and the pores are interconnected, by the principle of communicating vessels, the pressure in the whole phase will be uniform. No "mechanical" fluctuations are, therefore, identified within pore phase. If the sample is not additionally mechanically loaded, no fluctuations will arise. On the other hand, fluctuations can be estimated within the pores. In this case, fluctuations related to shear are zero. In the unsaturated case, different classes of pores may be subjected to different pressures. Additional complexity arises from the saturation state. Throughout this paper, we consider saturated media with unconnected

\subsection{Propagation of fluctuations in localization}

In contrast with homogenization procedure (bottom-up), localization procedure (top-down) consists in deriving the average mechanical fields in the microscopic scale from the information at the macroscopic scale. In the localization of mechanical fields in multi-scale materials, the fluctuations propagate through smaller scales. The average stresses at smaller scales tend to concentrate in stiffer phases and to diminish in less stiff phases.

Let the fluctuations of stress fields associated with a phase $i$ at a scale $s$ be $q_{i}^{s}$. At the macroscopic scale $s=0$ and the loading $\Sigma$ is applied on the REV. We assume that the local fluctuations $\left(q_{i}^{s}\right)_{\text {local }}$ associated with lower scales $s \geq 1$ are decorrelated from the fluctuations of all upper scales, being a function of only the average stresses $\langle\sigma\rangle_{j}^{s-1}$. The contribution of the higher scales to the fluctuations at a given scale $s \geq 1$ are expressed in terms of $\mathbb{B}_{j}^{s-1}: q_{j}^{s-1}:\left(\mathbb{B}_{j}^{s-1}\right)^{t}$, where $\mathbb{B}_{k}^{s-1}$ is the stress localization tensor of phase $j$ in which the domain of phase

and $Y$, the variance (or fluctuation) $q_{Z}$ of the sum $Z=Y+X$ is $q_{Z}=q_{Y}+q_{X}$. The total fluctuation at a scale $s>1$ is, therefore, expressed by:

$$
q_{i}^{s}=\left(q_{i}^{s}\right)_{l o c a l}+\mathbb{B}_{j}^{s-1} \otimes \mathbb{B}_{j}^{s-1}:: q_{j}^{s-1}
$$


Substituting recursively $q_{i_{s}}^{s-1}$ in the last equation, we obtain:

$$
q_{i}^{s}=\left(q_{i}^{s}\right)_{\text {local }}+\prod_{s_{s}=1}^{s-1}\left(\mathbb{B}_{i_{s}}^{s_{s}} \otimes \mathbb{B}_{i_{s}}^{s_{s}}\right):: q_{i_{0}}^{0}+\sum_{s_{s}=1}^{s-1}\left(\prod_{s_{t}=1}^{s_{s}}\left(\mathbb{B}_{i_{t}}^{s_{t}} \otimes \mathbb{B}_{i_{t}}^{s_{t}}\right)\right)::\left(q_{i_{s}}^{s_{s}}\right)_{\text {local }}
$$

where the product $\prod$ is defined in terms of tensor quadruple contraction "::". The subscript $i_{s}$ is equal to $i$ for a scale $s_{s}=s$ and equal to the corresponding index of the phase containing $\Omega_{i}^{s}$ at higher scales so that $\Omega_{i_{s}=i}^{s} \subset \Omega_{i_{s-1}}^{s-1} \subset \ldots \subset$ $\Omega_{i_{0}}^{0}$.

The bounds associated with one standard deviation in stresses are given by:

$$
\left(\sigma_{i}^{s}\right)^{ \pm}=\left(\prod_{i_{s}, r_{s}} \mathbb{B}_{i_{s}^{s}}^{s_{s}}\right): \Sigma \pm \omega_{i}^{s}
$$

where $\prod$ is defined in terms of tensor double contraction " : " and the standard

deviation $\omega_{i}^{s}$ can be estimated by $\left(q_{i, k l m n}^{0}\right)^{1 / 2}$ according to a given loading and direction associated with index $k l m n$.

Following this strategy to propagate the fluctuations, we ensure the normality of the distributions of stresses on all scales. A more precise way of propagating the fluctuations would be to apply a Monte-Carlo approach in which the distribution of mechanical fields in the higher scale is empirically propagated. In this case, the distribution of mechanical fields itself can be estimated at a given scale and the normality of the distribution is not assumed a priori. This procedure is much more computationally expensive than our proposition since, instead of one single computation of fluctuations, more than circa $10^{4}$ to $10^{5}$ computations would be required to properly describe the distribution of mechanical fields. We argue that our simplified way of accounting for the propagation of mechanical fields is consistent with the other simplifying assumptions adopted in homogenization/localization procedures, notably the ones related to the morphology of phases in each scale. Also, since we compute local fluctuations as a function of the average fields only, our estimations remain conservative with respect to other approaches. In other words, fluctuations propagating, as 
proposed here in a multiscale framework, are close to the minimal values expected in a composite due to the contrast of properties and volume fraction of constituent phases.

\subsection{Estimation of compressive mechanical strength}

The mechanical strength of composites can be estimated from the intraphase distribution of stresses associated with a (stress) failure criterion. Being stresses a tensorial quantity, it is necessary to deal with a multivariate distribution $\wp\left(\langle\sigma\rangle_{i}, q_{i}\right)$ of stress tensor in a given constituent phase $i$. For compressive strength, adopting Von Mises criterion, which corresponds to second invariant of stresses:

$$
\sigma_{V M}^{2}=\frac{1}{2}\left[\left(\sigma_{11}-\sigma_{22}\right)^{2}+\left(\sigma_{22}-\sigma_{33}\right)^{2}+\left(\sigma_{33}-\sigma_{11}\right)^{2}+6\left(\sigma_{23}^{2}+\sigma_{31}^{2}+\sigma_{12}^{2}\right)\right]
$$

the resulting distribution is 6 -variate, with each degree of freedom corresponding to one term in the right-hand side of the equation.

Let us assume that the stress components are random variables that are independent. The distribution of the difference of two random independent variables $X_{1}\left(\left\langle X_{1}\right\rangle, q_{1}\right)$ and $X_{2}\left(\left\langle X_{2}\right\rangle, q_{2}\right)$ is $X_{1-2}\left(\left\langle X_{1}\right\rangle-\left\langle X_{2}\right\rangle, q_{1}+q_{2}\right)$. Note that in the resulting distribution of the difference the variances are summed. The product of a random variable $X_{1}$ and a scalar $a$ verifies $a X_{1}\left(\left\langle X_{1}\right\rangle, q_{1}\right)=$ $X_{1}\left(a\left\langle X_{1}\right\rangle, a^{2} q_{1}\right)$. With these properties of independent random variables, the distributions of Von Mises stress can be computed from the Euclidean norm (or 2-norm) of a multivariate distribution with $k_{\chi}=6$ degrees of freedom accounting for each term of the right-most side of Eq. 29. The multivariate distribution of normally distributed random variables $\left\langle X_{i}\right\rangle^{2} / q_{i}$ is given by the noncentral chi-squared distribution whose PDF is:

$$
f_{\chi}\left(k_{\chi}, \lambda ; x\right)=\frac{1}{2} \exp \left(-\frac{x+\lambda}{2}\right)\left(\frac{x}{\lambda}\right)^{\frac{k_{\chi}}{4}-\frac{1}{2}} J_{\frac{k_{\chi}}{2}-1}(\sqrt{\lambda x})
$$


where $J_{v}(y)=(y / 2)^{v} \sum_{j=0}^{\infty} \frac{\left(y^{2} / 4\right)^{j}}{j ! \Gamma(v+j+1)}$ is the modified Bessel function of the first kind. The mean of noncentral chi-squared distribution is the sum of the degrees of freedom $k_{\chi}$ and non-centrality parameter $\lambda=\sum_{i=1}^{k_{\chi}}\left(\frac{\left\langle X_{i}\right\rangle}{q_{i}}\right)^{2}$. The variance of noncentral chi-squared distribution is $q_{\chi}=2\left(k_{\chi}+2 \lambda\right)$.

In a REV $\Omega$, a given phase $i$ with compressive strength $f_{c, i}$ is expected to fail in a uniaxial test if in a volume fraction $f_{c r}$ the von Mises stress exceeds $(2 / 3) f_{c, i}^{2}[18$. The compressive strength of the REV is therefore:

$$
f_{c, \Omega}=\min _{\forall i \subseteq \Omega}\left[\frac{f_{c, i} \sqrt{\frac{2}{3}}}{\sqrt{\frac{q_{\chi}}{k_{\chi}} F_{\chi}^{-1}\left(1-f_{c r}\right)}}\right]
$$

where $F_{\chi}^{-1}$ is the inverse of the Cumulative Distribution Function (CDF) of the noncentral chi-squared distribution $f_{\chi}$ in Eq. 30. The value of the critical volume fraction $f_{c r}$ must be chosen so that the strength of the composite $f_{c, \Omega}$ does not exceed the strength $f_{c, i}$ of the constituent phases $i$ [18].

\section{Results}

\subsection{Comparison with numerical simulations}

Figure 2 shows a comparison of our analytical estimates with 3D numerical simulation of Lavergne et al. [18 in a composite under uniaxial load in $x$-direction. The composite, mimicking a mortar, is composed of spherical inclusions embedded in a matrix. The Young moduli of the matrix and of the inclusions are 15 and $65 \mathrm{GPa}$, respectively; and the Poisson ratio of both phases is 0.2. Good agreement is observed between numerical and analytical results using MT scheme. For the matrix phase, we obtain the average value of $\sigma_{x x} / \sigma_{0}=0.724$ and the standard deviations $\omega_{x x} / \sigma_{0}=0.162$, $\omega_{y y} / \sigma_{0}=\omega_{z z} / \sigma_{0}=0.080, \omega_{x y} / \sigma_{0}=\omega_{x z} / \sigma_{0}=0.048$ and $\omega_{y x} / \sigma_{0}=0.056$. Numerical simulations seem to underestimate the the variability of stress distributions. This effect is expected since the evaluation of mechanical fields in numerical methods is generally made by averaging over finite size regions. Other 


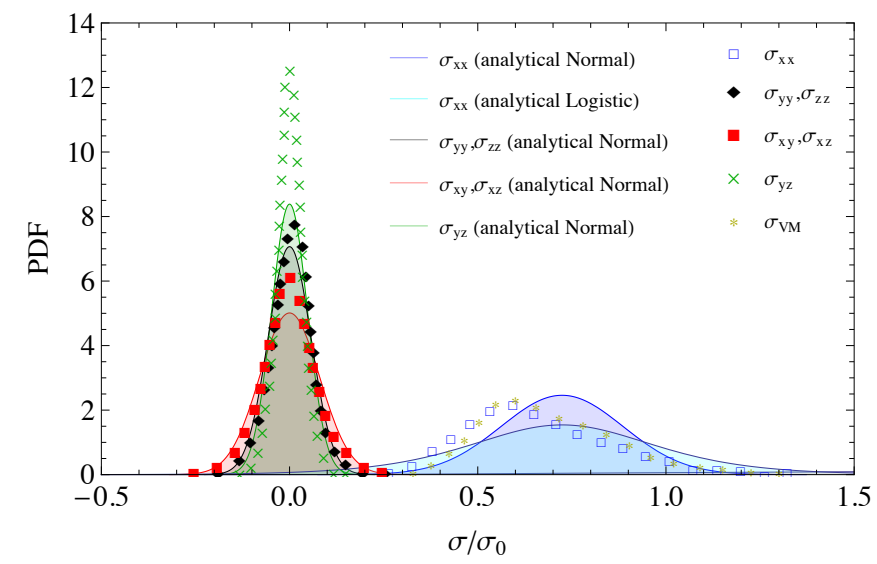

Figure 2: Comparison of analytical estimates of stress distribution with results from 3D numerical simulations of Lavergne et al. [18]. The composite, mimicking a mortar, is made of spherical inclusions embedded in a matrix. A uniaxial load is applied in the $x$-direction. The estimates are provided for the matrix only. The von Mises stress provided by the authors is also shown. In our estimations, we assume a normal distribution of stresses (unless stated otherwise).

distributions with larger kurtosis (e.g. logistic distribution) could also be envisioned to describe the statistical variability of stress fields.

We also performed simulations on numerical samples mimicking concrete at early-age. The inclusions have a Young modulus of $65 \mathrm{GPa}$ whilst the matrix has a increasing Young modulus: 5, 10 and $20 \mathrm{GPa}$ corresponding roughly to the very early-age (hours), mid-age (days) and late age (months), respectively. For all phases the Poisson ratio is 0.2. Analytical estimates are obtained using MT scheme. Figure 3(a) depicts the numerical sample with spherical inclusions considered in the finite elements simulations. The procedure for generating the microstructures is detailed in e.g. 47, 48, 46, 49, and has been applied to the study and estimation of various phenomena and physical properties. All geometries are obtained by randomly dispersing 4627 inclusions in a box, whatever their form, representing $50 \%$ in volume fraction (see [46]). The meshes (as well as the corresponding geometries) are periodic, allowing the application of periodic 
(a)

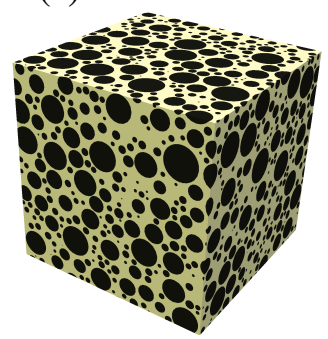

(b)

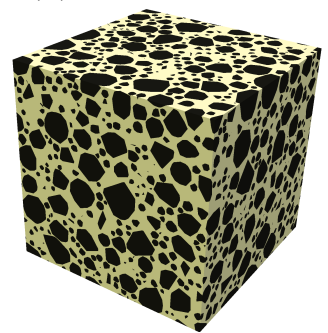

(c)

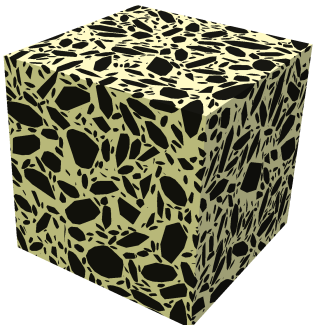

Figure 3: Numerical samples considered in 3D finite elements simulations: (a) spherical, (b) convex Voronoi polyhedral and (c) convex Voronoi polyhedral flattened (with aspect ratio of 3) inclusions [4].

stress boundary conditions. The number of tetrahedral finite elements ranges from $3.75 \times 10^{6}$ for Voronoi to $5.65 \times 10^{6}$ for spherical inclusions. All simulations are performed with the Cast3M FE code (www-cast3m.cea.fr). The stress field is then locally analyzed by computing the average stresses in all finite elements weighted by the corresponding element volume. Although we may argue that the results depend significantly on the mesh fineness and spatial resolution, it has been shown in [50] that the FE simulations were very close in terms of dispersion and macroscopic averaged values to FFT computations performed on the same geometries but with a considerably higher number of voxels. A uniaxial load is applied in $x$-direction. Figure 4 displays the numerical and analytical results of distribution of stresses in the matrix and inclusion at the three ages. Table 1 gathers the average and standard deviation of stresses according to analytical and numerical simulations. In general, analytical estimations return larger standard deviations of stresses when compared to numerical results. Analytical and numerical estimations of stress distributions for the matrix are in fair agreement in all ages and in all directions. On the other hand, within inclusions, the standard deviation of stresses, according to analytical estimates, are markedly larger than the standard deviation of stresses according to numerical results. With decreasing property contrast $\left(E_{\text {mat }} / E_{i n c} \rightarrow 1\right)$, numerical and analytical results become closer. 
Table 1: Average stresses in the direction of the load $\left\langle\sigma_{x x}\right\rangle / \sigma_{0}$ and standard deviations of stresses $\omega / \sigma_{0}$ according to analytical and numerical simulations.

\begin{tabular}{|c|c|c|c|c|c|c|c|c|}
\hline Phase & & $\left\langle\sigma_{x x}\right\rangle / \sigma_{0}$ & $\omega_{x x} / \sigma_{0}$ & $\omega_{y y} / \sigma_{0}$ & $\omega_{z z} / \sigma_{0}$ & $\omega_{x y} / \sigma_{0}$ & $\omega_{x z} / \sigma_{0}$ & $\omega_{y z} / \sigma_{0}$ \\
\hline Matrix & Analytical & 0.700 & 0.259 & 0.127 & 0.127 & 0.0690 & 0.0690 & 0.0911 \\
\hline \multirow[t]{3}{*}{$\left(E_{m a t} / E_{i n c}=0.0769\right)$} & Num. (Spherical incl.) & 0.60 & 0.340 & 0.102 & 0.101 & 0.114 & 0.113 & 0.0477 \\
\hline & Num. (Vor. incl.) & 0.544 & 0.331 & 0.0946 & 0.0949 & 0.112 & 0.112 & 0.0427 \\
\hline & Num. (Flat. incl.) & 0.536 & 0.305 & 0.0912 & 0.0908 & 0.112 & 0.110 & 0.0490 \\
\hline \multirow{4}{*}{$\begin{array}{c}\text { Matrix } \\
\left(E_{\text {mat }} / E_{\text {inc }}=0.154\right)\end{array}$} & Analytical & 0.732 & 0.215 & 0.101 & 0.101 & 0.0612 & 0.0612 & 0.0750 \\
\hline & Num. (Spherical incl.) & 0.654 & 0.299 & 0.0856 & 0.0852 & 0.101 & 0.100 & 0.0420 \\
\hline & Num. (Vor. incl.) & 0.611 & 0.286 & 0.0794 & 0.0797 & 0.0984 & 0.0988 & 0.0378 \\
\hline & Num. (Flat. incl.) & 0.616 & 0.265 & 0.0769 & 0.0761 & 0.0970 & 0.0952 & 0.0422 \\
\hline \multirow{4}{*}{$\begin{array}{c}\text { Matrix } \\
\left(E_{\text {mat }} / E_{\text {inc }}=0.308\right)\end{array}$} & Analytical & 0.791 & 0.160 & 0.0787 & 0.0787 & 0.0476 & 0.0476 & 0.0557 \\
\hline & Num. (Spherical incl.) & 0.743 & 0.225 & 0.0621 & 0.0618 & 0.0777 & 0.0771 & 0.0325 \\
\hline & Num. (Vor. incl.) & 0.719 & 0.215 & 0.0575 & 0.0578 & 0.0755 & 0.0758 & 0.0294 \\
\hline & Num. (Flat. incl.) & 0.728 & 0.200 & 0.0561 & 0.0557 & 0.0733 & 0.0721 & 0.0320 \\
\hline \multirow{4}{*}{$\begin{array}{c}\text { Inclusion } \\
\left(E_{\text {mat }} / E_{\text {inc }}=0.0769\right)\end{array}$} & Analytical & 1.300 & 0.791 & 0.387 & 0.387 & 0.214 & 0.2140 & 0.277 \\
\hline & Num. (Spherical incl.) & 1.40 & 0.289 & 0.106 & 0.106 & 0.122 & 0.121 & 0.0657 \\
\hline & Num. (Vor. incl.) & 1.470 & 0.402 & 0.133 & 0.132 & 0.161 & 0.161 & 0.0794 \\
\hline & Num. (Flat. incl.) & 1.470 & 0.488 & 0.162 & 0.161 & 0.192 & 0.188 & 0.104 \\
\hline \multirow{4}{*}{$\begin{array}{c}\text { Inclusion } \\
\left(E_{\text {mat }} / E_{\text {inc }}=0.154\right)\end{array}$} & Analytical & 1.268 & 0.519 & 0.252 & 0.253 & 0.149 & 0.149 & 0.181 \\
\hline & Num. (Spherical incl.) & 1.35 & 0.210 & 0.0767 & 0.0763 & 0.0897 & 0.0888 & 0.0485 \\
\hline & Num. (Vor. incl.) & 1.390 & 0.282 & 0.0924 & 0.0920 & 0.113 & 0.113 & 0.0559 \\
\hline & Num. (Flat. incl.) & 1.390 & 0.345 & 0.108 & 0.106 & 0.133 & 0.130 & 0.0687 \\
\hline \multirow{4}{*}{$\begin{array}{c}\text { Inclusion } \\
\left(E_{\text {mat }} / E_{\text {inc }}=0.308\right)\end{array}$} & Analytical & 1.209 & 0.286 & 0.141 & 0.141 & 0.0851 & 0.0851 & 0.0995 \\
\hline & Num. (Spherical incl.) & 1.260 & 0.123 & 0.0452 & 0.0450 & 0.0536 & 0.0531 & 0.0294 \\
\hline & Num. (Vor. incl.) & 1.28 & 0.162 & 0.0527 & 0.0527 & 0.0651 & 0.0650 & 0.0324 \\
\hline & Num. (Flat. incl.) & 1.270 & 0.203 & 0.0593 & 0.0583 & 0.0762 & 0.0747 & 0.0377 \\
\hline
\end{tabular}



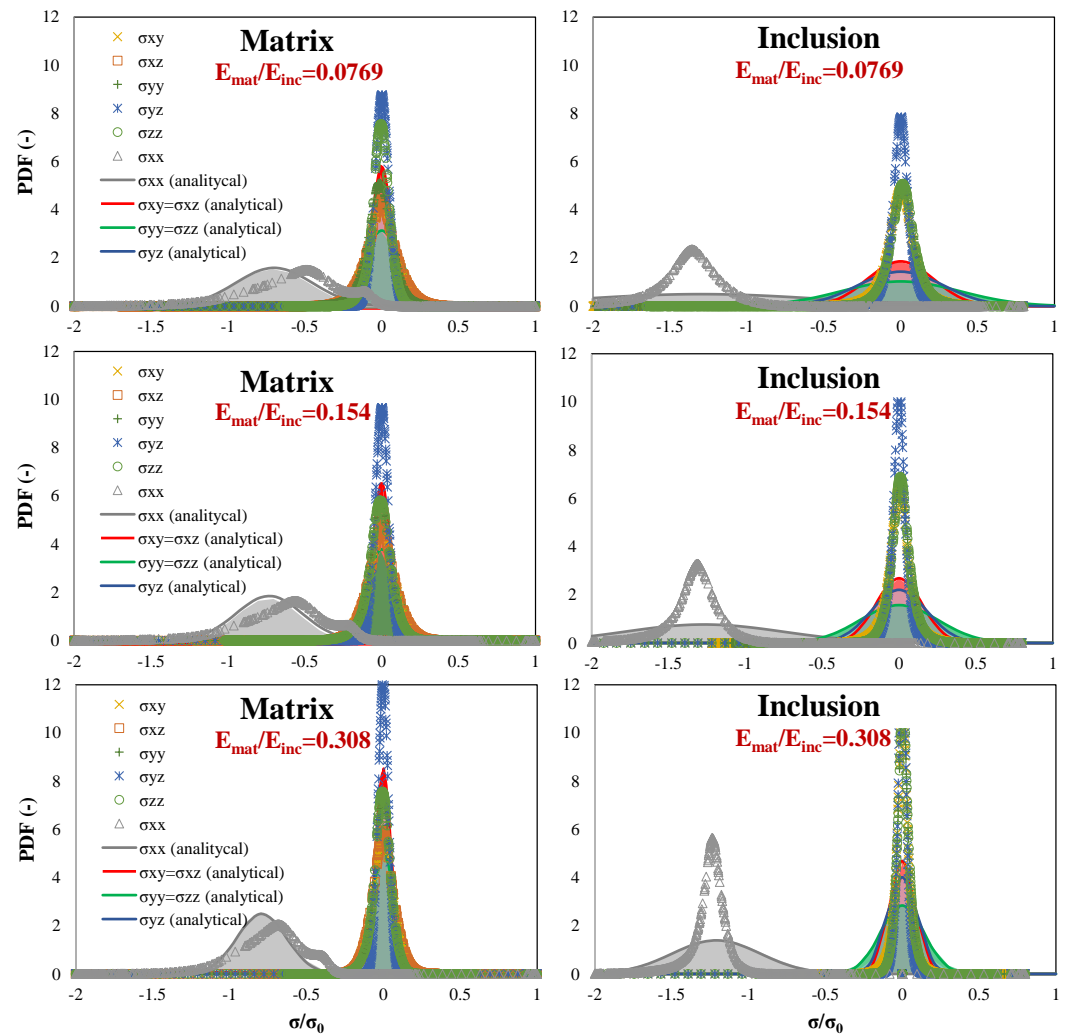

Figure 4: Comparison of analytical estimates of stress distribution in matrix and inclusions with results from 3D finite elements simulations. Only the numerical sample with spherical inclusions is considered for finite element simulations. The matrix depicts an ageing mortar with increasing Young modulus $E_{m a t}=5,10$ and 20 GPa corresponding to the very earlyages, few days of age and late ages, respectively. The Young modulus of the coarse aggregate is $E_{i n c}=65 \mathrm{GPa}$; the Poisson ratio of all phases is 0.2 . A uniaxial load is applied in $x$-direction. The analytical estimations are shown assuming a normal distribution of stresses. 
Additionally, the effect of coarse aggregate shape is considered in Figure 5 for Figure 3(b) and (c) depicts the numerical samples with convex Voronoi polyhedral (b) and flattened (with aspect ratio of 3) convex Voronoi polyhedral (c) as considered in the FE simulations. The PDF is shown in logarithmic scale in order to highlight the high kurtosis of numerical results regarding $x$-direction. numerical results. Compared to the case with spherical inclusions, the stress distribution curves in the composite with Voronoi inclusions are broadened. As expected, the flattened polyhedral inclusions lead to broader distributions of stresses within the aggregates considering all directions. Note however that, with 375 flattened polyhedral inclusions, the distribution of stresses within the matrix is less broad than for spherical inclusions. The results in 5 show that the analytical estimations of standard deviations can capture even the stress distribution associated with flattened inclusions embedded in a matrix.

With the results in this section, however, it is not possible to identify which technique, analytical or numerical simulations, yields estimations of standard deviations of stresses in composites that are closer to the variability of stresses in "real" composites. In both cases, specific underlying assumptions may impact the estimates. Remarkably, both techniques lead to results of variability of stresses that are on the same order of magnitude. This observation corroborates of stresses in composites.

\subsection{Application to estimation of fluctuations within $C-S-H$}

A second application is to study a simple example of a porous material with saturated pores. We compare estimations of micro- and macro-isotropic MT and SC schemes as presented in Section 2.2. These simple morphologies are used to represent C-S-H according to different packing densities. C-S-H is often reported to occur in two specific average packing densities generally called Low Density (LD) and High Density (HD) products. According to Constantinides et 

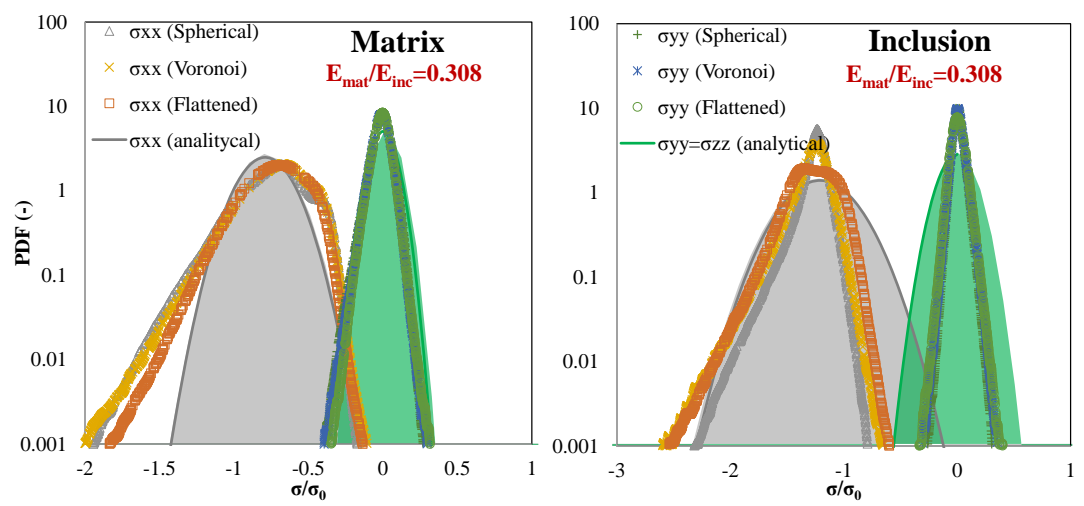

Figure 5: Effects of inclusion shape: comparison of analytical estimates of stress distributions in matrix and inclusions with results from 3D finite elements simulations on numerical samples with three different inclusion shapes. The Young moduli of the matrix and inclusion are $E_{m a t}=20 \mathrm{GPa}$ and $E_{i n c}=65 \mathrm{GPa}$, respectively; the Poisson ratio of all phases is 0.2 . A uniaxial load is applied in $x$-direction. The analytical estimations are shown assuming a normal distribution of stresses.

al. 51, LD and HD C-S-H present an average packing density of 0.64 and 0.76 , respectively. Undrained conditions with closed pores are assumed so that the fluctuations within pores can be assessed. In this case, since the bulk modulus of water $(2.18 \mathrm{GPa})$ is taken into account in the homogenization, the percolation threshold of self-consistent scheme is close to 0.4. In all cases, the solids grains of C-S-H are assumed homogeneously distributed over the REV.

The average stresses and the corresponding standard deviations of C-S-H according to the packing density are shown in Figure 6. Both SC and MT estimates are displayed and the fluctuations are computed in the principal direction of the loading. At the left, the Probability Density Functions (PDF) are shown for both solids and pores. Hereafter, in PDF plots, we assume normal distribution of stresses. MT and SC return similar estimations of fluctuations for packing densities above 0.7. The estimations of the fluctuations of stresses of LD C-S-H with SC scheme are slightly larger than with MT scheme. Regarding the unconnected pores, the pores in MT estimations are subjected to larger average stresses than in SC estimations. The fluctuations are larger within LD 

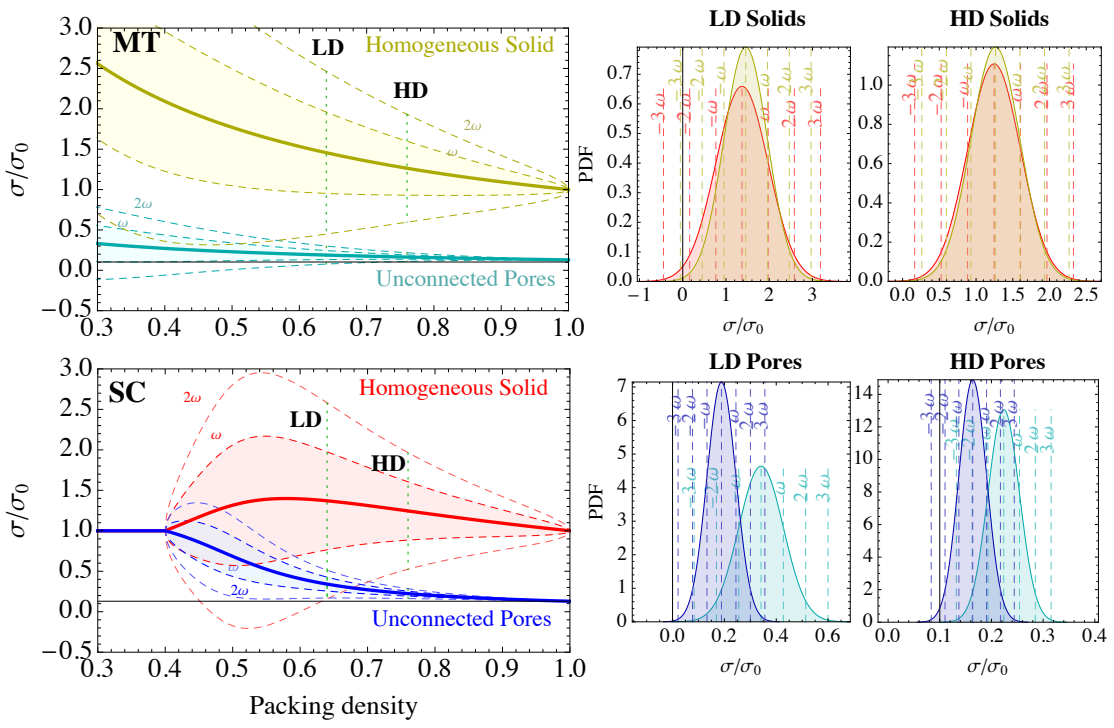

Figure 6: Stress fluctuations within 'undrained' C-S-H according to packing density for a hydrostatic load (left). At right, the PDF at the packing densities corresponding to HD and LD C-S-H, (0.76 and 0.64, respectively) assuming normal distribution.

pores as computed by MT scheme. For both estimates, LD solid C-S-H and corresponding pores are subject to larger fluctuations compared to HD C-S-H. For a hydrostatic loading, with SC scheme the maximum fluctuations in the solid arise at a packing density of circa 0.55 and in the pore at a packing density of 0.5. With MT scheme, the fluctuations increase with the packing density tending to 0 . Accounting for two standard deviations is enough to observe opposite sign loading within a given phase, i.e. traction if $\sigma_{0}$ is compression and vice-versa, for low packing densities (below 0.65 for solids in SC scheme and 0.65 for pores in MT scheme). The estimations of the standard deviation of stresses computed with both schemes in HD and LD C-S-H have the order of magnitude of half the load applied on the bulk material.

As can be observed in the formulation presented in Section 3. fluctuations are load dependent. Figure 7 shows the PDF of stresses in the direction 11 in 
solids for LD C-S-H according to four different loads. Hydrostatic, uniaxial, biaxial and pure shear are considered. The base of comparison of these different loads is the maximum principal stresses in all cases being 1 . On this criterion, anisotropic loads exhibit larger fluctuations with pure shear load depicting the largest ones among the loads considered in the figure. Further interpretations of the load-dependence of fluctuations must be made with care since comparing tensorial quantities is not a trivial task. Other criteria could be defined in order to establish a comparison; for example, the trace of the tensor of the principal stresses or a criterion on energy. Moreover, the fluctuations in directions that are not the principal directions could also be compared. An interesting finding is that, in spite of the differences due to load type, the fluctuations in the tested cases are all of the same order of magnitude for loads with a maximum principal stress is equal to 1 .

Estimations of fluctuations in a mesoscopic scale of C-S-H can also be computed according to a distribution of packing densities over a REV.

Distributions of packing densities at mesocale of C-S-H, obtained from experiments and simulations, are reported by Ioannidou et al. [52. We consider the experimental samples "S1" (made with oil-well cement, class G and quartz hydrated at $\mathrm{w} / \mathrm{c}=0.43$ ) and "S3" (made with ordinary Portland cement type I (OPC CEM-I) and hydrated at room temperature with $\mathrm{w} / \mathrm{c}=0.35)$ obtained by nanoindentation, provided by these authors. Note that Constantinides et al. 51] used a cement similar to the one of S3 samples and also provided the distribution of packing densities in C-S-H mesoscale. The corresponding distributions of volume of the zones with a given packing density are shown in Figure 8 (top). Each packing density in 8 (top) is considered as a phase whose mechanical properties are obtained by homogenization as in Figure 6 with SC scheme. Then, the regions with a given packing density are treated again as homogeneous equiaxed grains organized in a polycristal-like microstructure. We compute the fluctuations associated with each packing density at this mesoscale as displayed in Figure 8 (bottom). In both curves, the fluctuations gradually increase with the stiffness (or packing density) of a zone irrespectively of its 


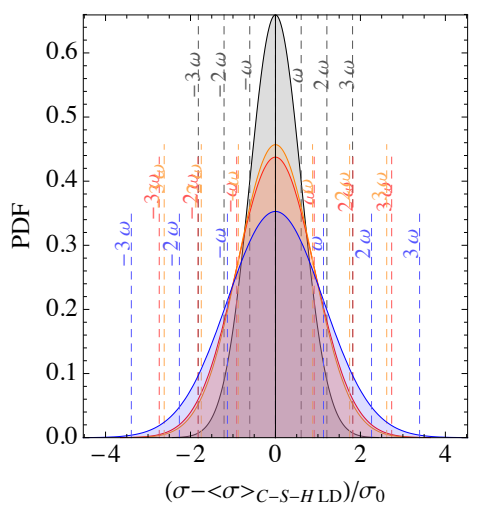

$$
\begin{array}{ll}
\text { - Hydrostatic: } & \sigma / \sigma_{0}=\left(\begin{array}{lll}
1 & 0 & 0 \\
0 & 1 & 0 \\
0 & 0 & 1
\end{array}\right) \\
\text { — Uniaxial: } & \sigma / \sigma_{0}=\left(\begin{array}{lll}
1 & 0 & 0 \\
0 & 0 & 0 \\
0 & 0 & 0
\end{array}\right) \\
\text { घ Biaxial: } & \sigma / \sigma_{0}=\left(\begin{array}{lll}
1 & 0 & 0 \\
0 & 1 & 0 \\
0 & 0 & 0
\end{array}\right) \\
\text { - Pure Shear: } & \sigma / \sigma_{0}=\left(\begin{array}{lll}
0 & 1 & 0 \\
1 & 0 & 0 \\
0 & 0 & 0
\end{array}\right)
\end{array}
$$

Figure 7: Probability Density Function (PDF assumed to be Gaussian) of stress fluctuations in LD C-S-H depending on loading type in 11 direction. For all loads, the maximum principal stress is 1 . 
volume fraction. In the range of packing densities shared by both cases, a zone the overall stiffness is larger in this case.

The estimations provided in this section account neither for the anisotropy and disorder [53] which must increase the heterogeneity of mechanical fields in C-S-H nor for aspects related to confinement of water which may lead to solid or glassy-like behaviours of pore phase [54. Additionally, the simplified representations, often adopted in homogenization-based studies, may not capture the complexities of the assembly of hydration products as reported by nanoindentation investigations strategies coupled with chemical techniques [55, 56, 57].

\subsection{Application to $C B M$}

\subsubsection{Multiscale representation of $C B M$}

The multiscale representation of CBM microstructure used here is similar to the one proposed in [10] and used previously by other authors [58, 9]. Twocoated sphere morphology based on GSC scheme is adopted to represent cement paste and mortar. At cement paste scale, the hydrating clinker particle is embedded in a high density (HD) products layer, which is embedded in a low density (LD) products layer. Both product layers are composed of the different products resulting from cement hydration processes; the difference is the presence of HD C-S-H in HD layer and LD C-S-H in LD layer. In this paper, we compare estimations of the effective properties of hydration product layers (i.e. about the separability of the scales in the sub-problems at cement paste scale can be found in $[9$.

At mortar scale, a homogeneous cement paste embeds ITZ, which in turn embeds sand particles. GSC scheme is used to obtain the homogenized properties of mortar. As mentioned, estimations of the effective properties of ITZ from MT and SC schemes are provided. At concrete scale, coarse aggregates are embedded in a homogeneous mortar matrix. ITZ around coarse aggregates is not accounted for since its volume is negligible with respect to the ITZ of fine 

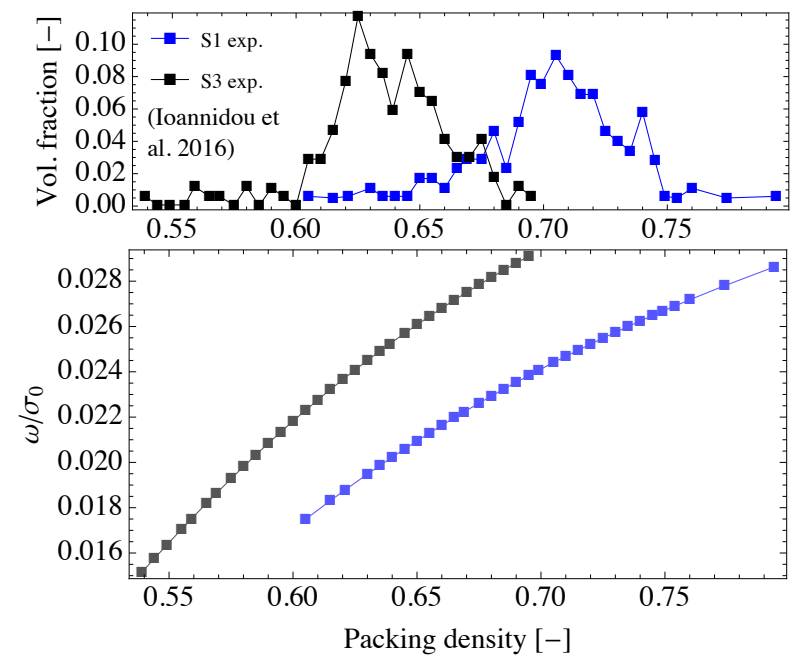

Figure 8: Stress fluctuations for a polycrystal-like composite with equiaxed inclusions presenting different packing densities. An hydrostatic stress load is applied. The distribution, in terms of volume fraction, of packing densities follow the sets S1 and S3 of Ioannidou et al. 52 


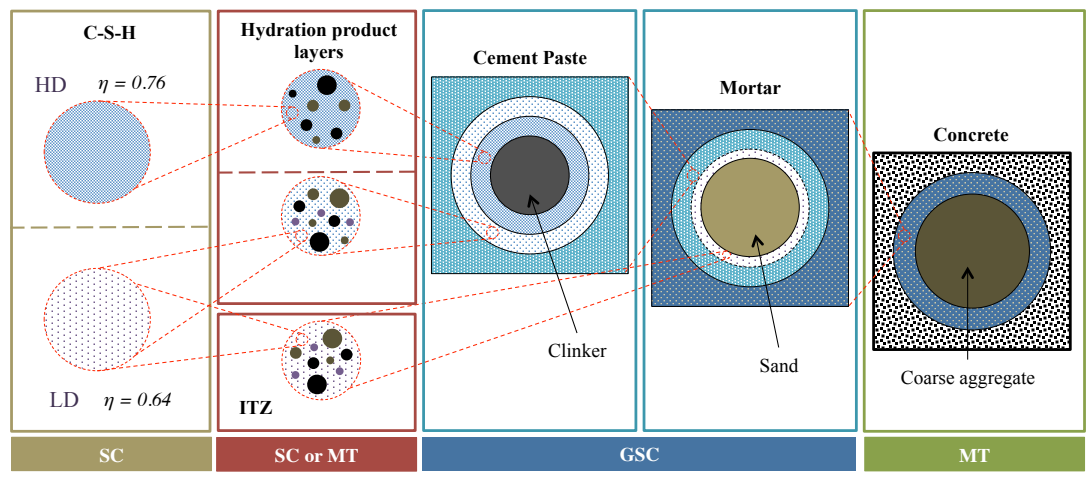

Figure 9: Representation of the multiscale character of CBM

aggregates. MT scheme is used to obtain the effective properties of concrete. The assumption of the separability of cement paste and mortar scales seems adequate since fine aggregates are in general larger than $100 \mu \mathrm{m}$, which is reported to be the maximum size of crystalline inclusions in cement paste [59. Concerning the separability of mortar and concrete scales, coarse aggregates size can be considered about one order of magnitude bigger than fine aggregates.

The kinetics of hydration processes is modelled using the approach detailed in 60. The evolution of volume fractions at cement paste scale is estimated by means of Tennis and Jennings [61] stoichiometric balance equations with slight modifications [10] so that HD product is formed after the peak in heat flux curves. We divide hydration products in LD and HD layers proportionally to LD and HD volume fractions as in Honorio et al. [6].

In the scenarios with ITZ, a part of the products in LD layer is discounted for to constitute the ITZ. This aspect introduces a dependency of the composition of LD layer on ITZ composition and size. Following a previous work [6], we assume ITZ is $10 \mu \mathrm{m}$ thick, and its total volume is computed taking into account the interpenetration of neighbouring ITZ.

The composition of the cement (CEM I) considered is as follows in terms of mass fractions: $\mathrm{C}_{3} \mathrm{~S}=0.64, \mathrm{C}_{2} \mathrm{~S}=0.231, \mathrm{C}_{3} \mathrm{~A}=0.015, \mathrm{C}_{4} \mathrm{AF}=0.020, \mathrm{C} \overline{\mathrm{S}} \mathrm{H}_{2}$ $=0.02$ and $\mathrm{CaCO}_{3}=0.035$. The particle size distributions of cement and sand 

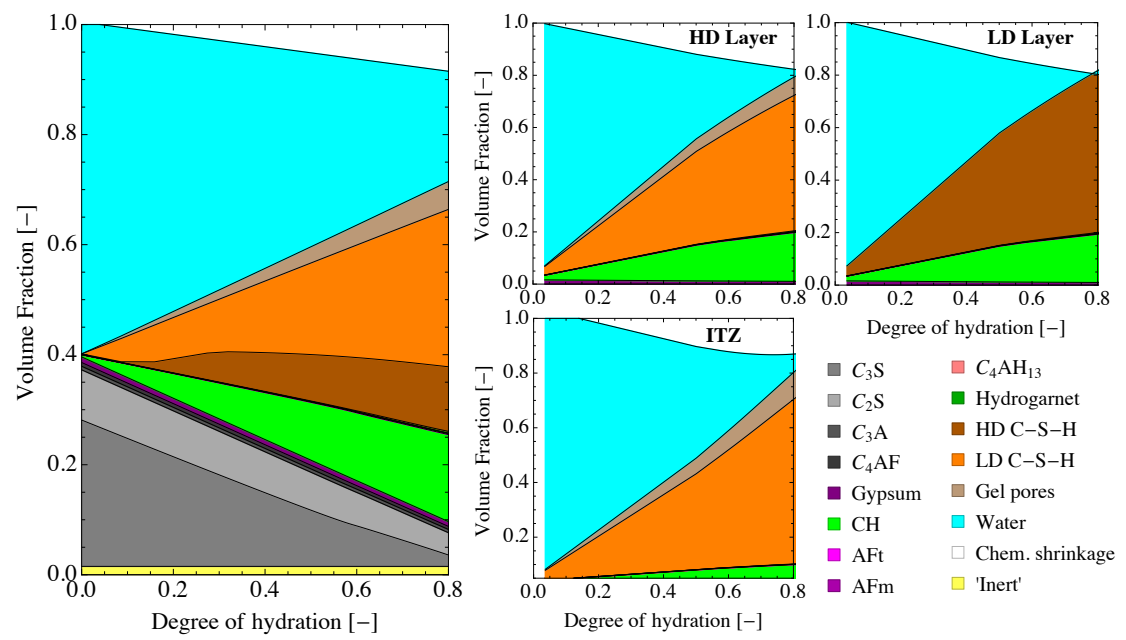

Figure 10: Evolution of volume fractions of clinker minerals and hydrate products with respect to the degree of hydration. Cement composition as in [6] with $\mathrm{w} / \mathrm{c}=0.5$. At left, repartition of cement hydration products in HD and LD layers as well as ITZ [6]. The repartition in HD and LD layers follows the relative volume fraction of HD and LD C-S-H. ITZ is assumed to be $10 \mu \mathrm{m}$ thick

are detailed in Honorio et al. 6]. Figure 10 (left) displays the evolution of the volume fraction of phases at cement paste scale for $\mathrm{w} / \mathrm{c}=0.5$. On the right, the repartition of hydration products within HD, LD and ITZ layers is shown.

\subsubsection{Homogenization}

A first step of homogenization is carried out in order to obtain the effective properties at each scale detailed in the last section. The elastic properties of phases are gathered in Table 2. Figure 11 shows the homogenized bulk and shear modulus at cement paste, ITZ, mortar and concrete scales. The estimations of the effective properties of the layer of hydration products are 
Table 2: Elastic properties of phases

\begin{tabular}{|c|c|c|c|}
\hline Phase & E [GPa] & $\nu[-]$ & Ref. \\
\hline Clinker & 140 & 0.3 & $62]$ \\
C-S-H HD & 29.4 & 0.24 & {$[1]$} \\
C-S-H LD & 21.7 & 0.24 & {$[1]$} \\
$\mathrm{CH}$ & 38 & 0.305 & $63]$ \\
$\mathrm{AFt}$ & 22.4 & 0.25 & $64]$ \\
$\mathrm{AFm}$ & 42.3 & 0.324 & $64]$ \\
$\mathrm{C}_{3}(\mathrm{~A}, \mathrm{~F}) \mathrm{H}_{6}$ & 21.7 & 0.24 & $65]$ \\
$\mathrm{C}_{4} \mathrm{AH}{ }_{1} 3$ & 25 & 0.25 & $6 \underline{65}$ \\
$\mathrm{C}_{\bar{S}} \mathrm{H}_{2}$ & 45.7 & 0.33 & 65 \\
\hline Sand (limestone) & 74.5 & 0.2 & 66 \\
Coarse aggregate (limestone) & 74.5 & 0.2 & 66 \\
\hline
\end{tabular}

computed according to MT and SC schemes. The resulting effective properties of cement paste are shown in the Figure 11. The same is done for ITZ. Estimations without accounting for ITZ are also provided; in this case, only the scenario with SC homogenization of product layers is considered. The material is stiffer in the absence of ITZ. We considered the pores as saturated and unconnected, i.e. water is the only fluid inside the pores and behaves similarly to a solid. With that assumption, one can assess the fluctuations within pores. The cusp points in SC estimations are due to percolation threshold associated with this scheme.

\subsubsection{Fluctuations in elasticity}

In this section, we compute the fluctuations of stress fields without accounting for the presence of transformation fields. The statistical variability of stresses is presented following the three scenarios presented in the last section regarding homogenization of hydration products at cement paste and ITZ scales (with MT or SC schemes) as well as a scenario not accounting for an ITZ (SC scheme for the layers of hydration products). Figure 12 shows the average stresses and standard deviation of stresses at concrete scale in which coarse aggregate are embedded in a mortar matrix. A hydrostatic loading $\sigma_{0}$ is applied and the 

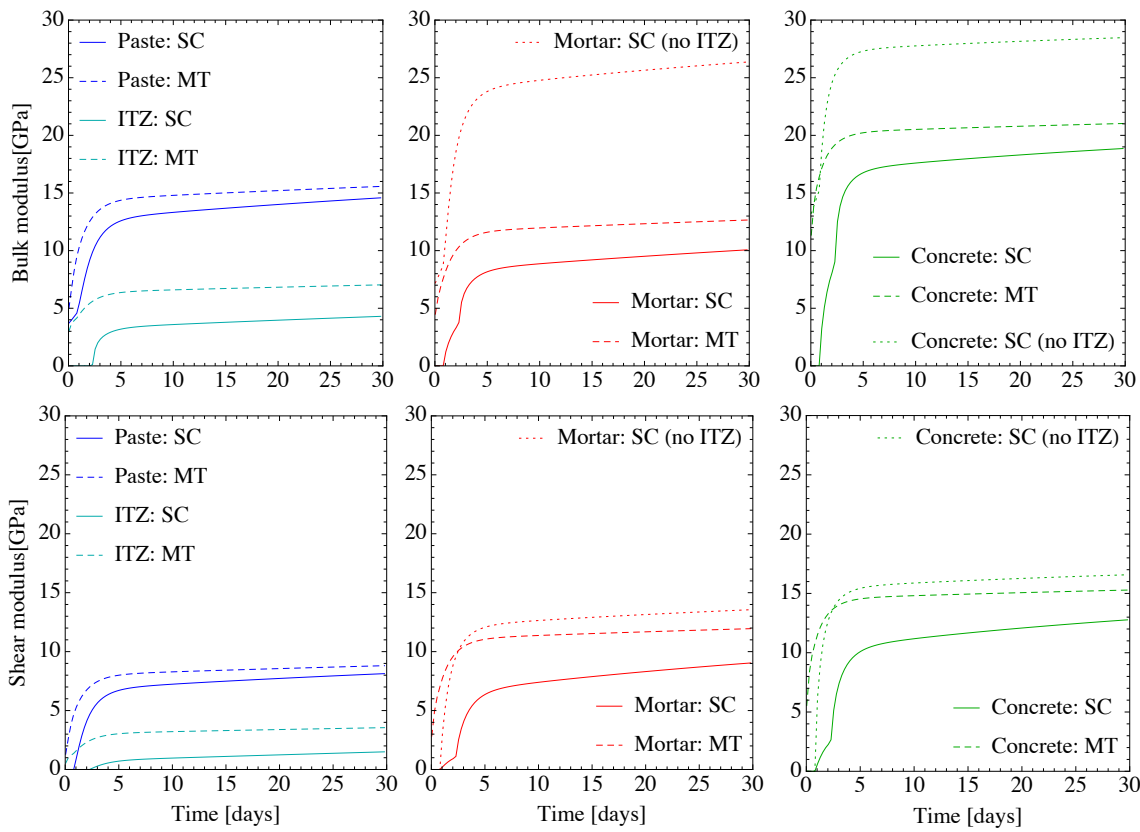

Figure 11: Homogenization of elastic properties at cement paste, ITZ, mortar and concrete scales. Scenarios with and without accounting for ITZ at mortar scale and concrete scale. ITZ of coarse aggregates are not considered in all cases. SC (resp. MT) stands for estimations of the effective properties of product layers (LD layer, HD layer and ITZ) with SC (resp. MT) scheme. In Honorio 67] the scenario with ITZ and MT scheme is compared with experimental results of samples with the considered cement and $\mathrm{w} / \mathrm{c}=0.5$. 
fluctuations are computed in one of the principal directions of the load. The mortar is stiffer in the scenario without ITZ. So, the contrast between coarse aggregates and mortar is lesser than in the scenarios with ITZ. This translates in fluctuations being smaller in the scenario without ITZ. In the scenarios accounting for the ITZ, similar standard variations are observed irrespective of the schemes used to homogenize hydration products. At early-age, the fluctuations are larger than in late ages. In terms of coefficient of variation (COV), defined as the ratio between the maximum standard deviation and the average at a given time, the difference between stiffer and softer phases are greater at the very early-ages and becomes smaller at late ages.

Figure 13 displays the average stresses and standard deviation of stresses at mortar scale for the hydrostatic loading $\sigma_{0}$ applied at concrete scale. The fluctuations are larger in the presence of ITZ. Akin to concrete scale, the fluctuations are larger in the stiffer phases, which, in this example, are the aggregates. It is noteworthy that stresses localized within the ITZ are smaller than the macroscopic applied stress.

The average stresses and standard deviation of stresses at cement paste paste scale, as defined in Figure 9 in terms of concentric spheres, are shown in Figure 14. The clinker is the most loaded phase since it is stiffer than the homogenized layers of hydration products. At this scale, the effect of the propagation of fluctuations, as discussed in Section 3.2, is clear with fluctuations of stresses within clinker being the largest among all phases across each one of the scales considered. The average stresses and standard deviation of stresses at the layers are quite similar, with HD layer presenting slightly larger fluctuations of stresses. In the following, we retain HD layer as representative of stress states in hydration products in general.

Considering only HD layer, the average stresses and standard deviation of stresses at hydration products scale is displayed in Figure 15 . For the sake of readability, only $\mathrm{C}-\mathrm{S}-\mathrm{H} \mathrm{HD}$, aFm (the most stiff phase at this scale), $\mathrm{CH}$ and saturated pore phases are shown. The estimations of the averages stresses are notably dependent on the homogenization scheme adopted. In the comparison 

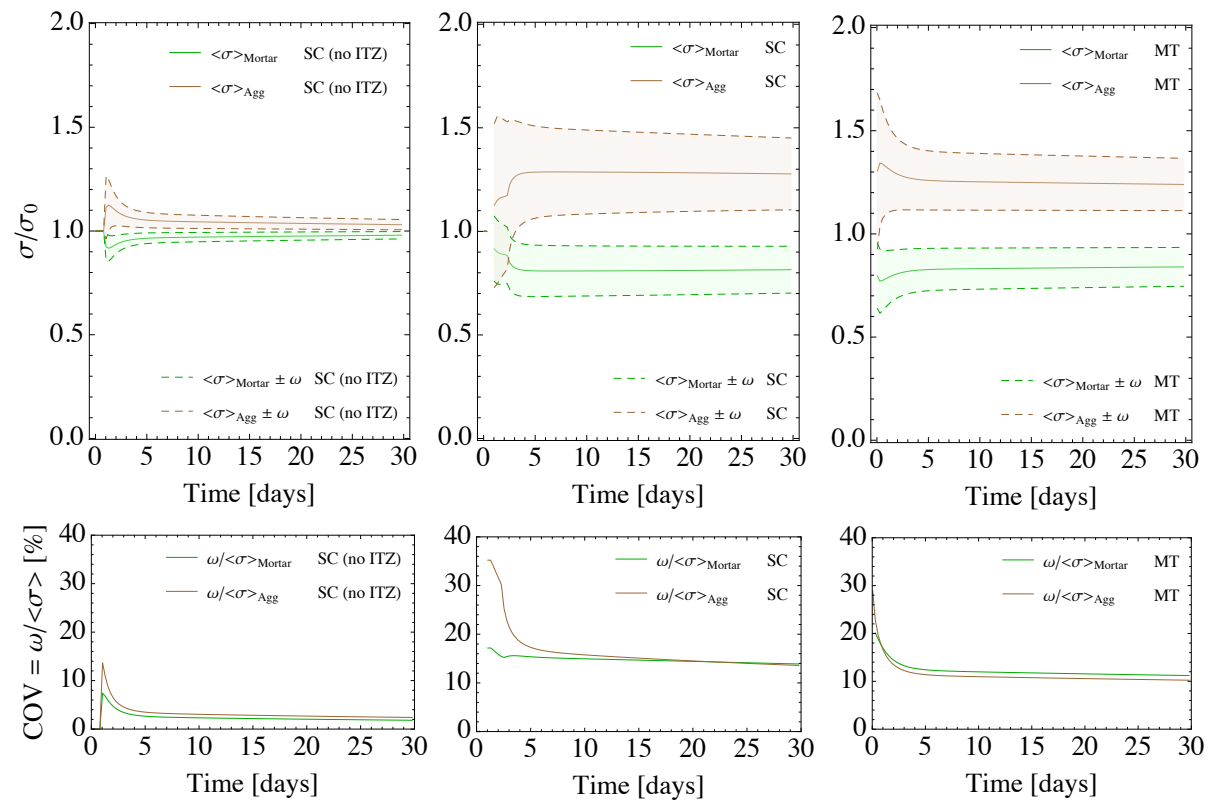

Figure 12: Average stresses and standard deviation of stresses at concrete scale for a hydrostatic loading $\sigma_{0}$ at concrete scale. Three scenarios (MT, SC and SC without ITZ) defined with respect to the homogenization of hydration products in cement paste and ITZ, as shown in Figure 11, are considered. The coefficient of variation (COV) defined as the ratio between the maximum standard deviation and the average (at a given time) is also provided. 

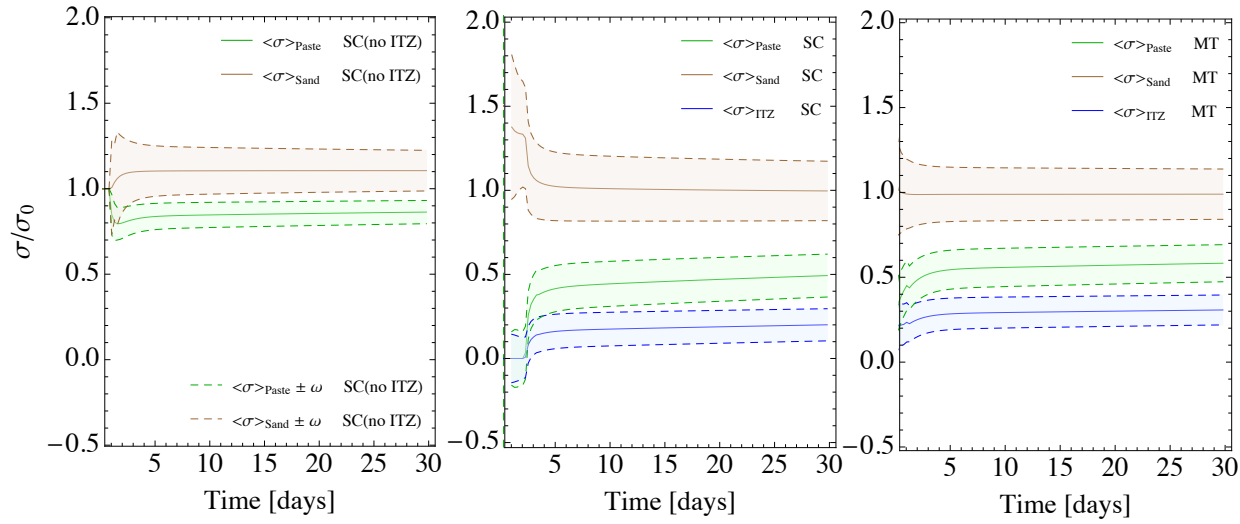

Figure 13: Average stresses and standard deviation of stresses at mortar scale for a hydrostatic loading $\sigma_{0}$ applied at concrete scale. 

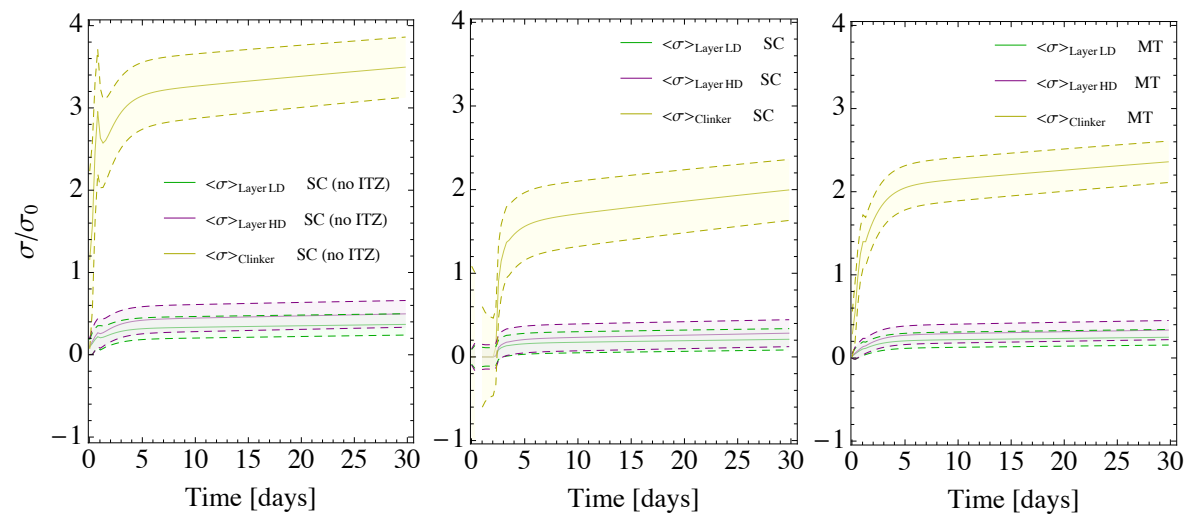

Figure 14: Average stresses and standard deviation of stresses at cement paste and hydration products scales for a hydrostatic loading $\sigma_{0}$ at concrete scale.

between SC scenarios with and without ITZ, these average stresses significantly differ since the presence of ITZ affects the averaging computation in localization process. Note, however, that both SC estimations (i.e. accounting or not for the ITZ) show, as expected, similar fluctuations of stresses. Again, the stiffer phase present larger fluctuations of stresses. In the scenarios with ITZ, with one standard deviation one may observe opposite sign loading in all phases (i.e. traction if $\sigma_{0}$ is compression and vice-versa). This effect is more pronounced with SC estimations. Except for the very early-age (before 1 day) in which percolation effects may disturb the results, the standard deviation of stresses increases with age.

A variety of aggregates with different mechanical properties can be used in concretes. Since fluctuations are a function of the stiffness of the phases, we recompute the average stresses and standard deviations of stresses at concrete 

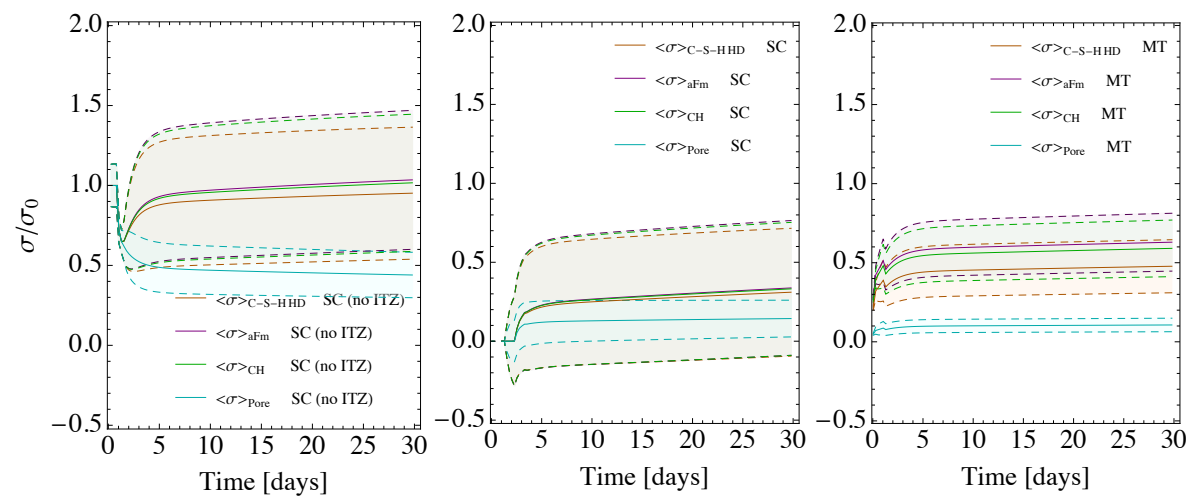

Figure 15: Average stresses and standard deviation of stresses at hydration products scales within HD layer for a hydrostatic loading at concrete scale. 
scale (as in Figure 12, but only with MT scenario) considering three classes of aggregates 68](pp. 93-94) according to their average Young moduli $E_{A g g}$ :

- Low porosity aggregates (e.g. granite and basalt) with Young moduli ranging from 70 to $140 \mathrm{GPa}\left(E_{A g g}=105 \mathrm{GPa}\right)$;

- High porosity aggregates (e.g. sandstones and limestones) with Young moduli ranging from 21 to $49 \mathrm{GPa}\left(E_{\text {Agg }}=35 \mathrm{GPa}\right)$; $\left(E_{\text {Agg }}=17.5 \mathrm{GPa}\right)$.

In all cases, we adopt a Poisson ratio of 0.2. The results are gathered in Figure 16. The fluctuations of stresses increase with the stiffness of the aggregates or more precisely, with the contrast between mortar and aggregates. The fluctuations in the concrete with lightweight aggregates are circa 4 times inferior to the fluctuations in concrete with low porosity aggregates. Thus, it would be expected that the onset of damage in concretes with stiffer aggregates is more likely than in concretes with aggregates with stiffeness close to mortar's (provided that the aggregates have a similar shape, size and surface texture in both cases). It does not mean, however, that concretes with less contrast between aggregate and mortar stiffnesses would be more resistant since aggregates with properties close to mortar's are more susceptible to damage per se. With lightweight aggregates, the mortar becomes the most loaded phase after few days. In this case, the failure mode may be affected as highlighted in the literature 69].

\subsection{Estimations of compressive strength of cement paste}

Figure 17 displays a comparison of analytical estimates of compressive strength of cement pastes against experimental data. The estimations are made at hydration layer scales, as in Figure 14. We follow the formulation presented in Section 3.3 considering both SC and MT schemes for homogenizing each hydration products' layer. At this scale, the adopted intrinsic compressive strength 

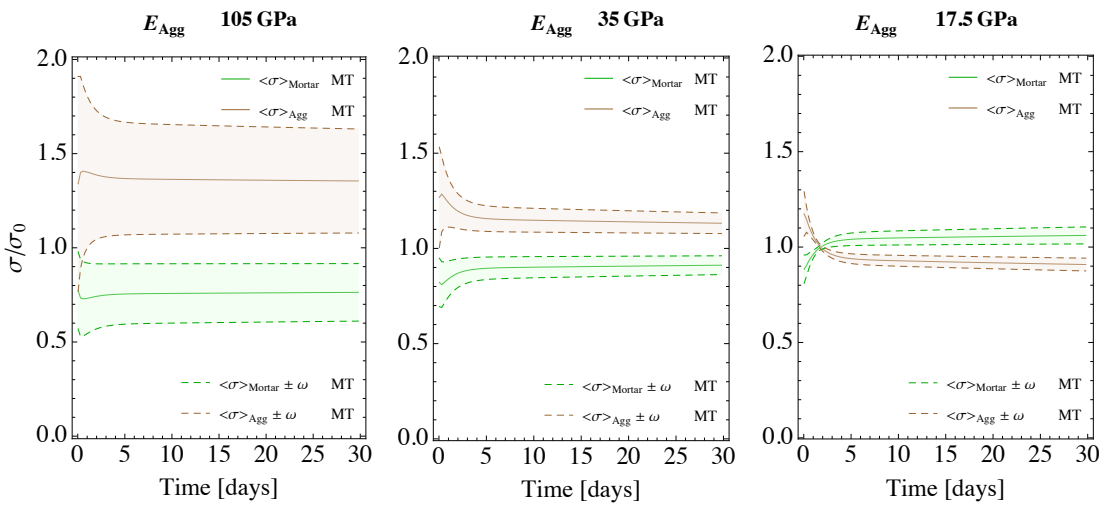

Figure 16: Average stresses and standard deviation of stresses according to different types of aggregates at concrete scale for a hydrostatic loading $\sigma_{0}$ at the same scale. Average Young modulus corresponding to low porosity $\left(\mathrm{E}_{A g g}=105 \mathrm{GPa}\right)$ and high porosity $\left(\mathrm{E}_{\text {Agg }}=35\right.$ $\mathrm{GPa})$ natural aggregates as well as lightweight aggregates $\left(\mathrm{E}_{A g g}=17.5 \mathrm{GPa}\right)$ are considered according to the MT scenario (with ITZ). 
of hydrates is $f_{c, 0}=141.5 \mathrm{MPa}$ [4]. We consider various values of the critical volume fraction $f_{c r}$. As in Lavergne et al. [18, values of $f_{c r}$ nearing 0.1 return reasonable estimations of compressive strength. Larger values of $f_{c r}$ may lead to estimations of the strength of a composite that are higher than the strength of the contituent phases.

The results in Figure 17 show that the estimations of strength are schemedependent, notably at early-ages. Hydration products' layer homogenized by SC scheme provides better estimations at the very early-ages than with MT scheme. It must be noted, however, that at the very early-age, the assumption of spherical (or equiaxed) inclusions and isotropy of phases seem not to be enough to capture the strength of the cement paste. This aspect was observed by Pichler et al. [0], which propose acicular inclusions to model hydrates in cement paste scale. Further investigations could also cope with higher fluctuations coming from the anisotropy of inclusions (not necessarily non-spherical ones). Our results, however, indicate that these simplifying assumptions of spherical (or equiaxed) inclusions and (micro-)isotropy are enough to capture the compressive strength of cement paste at late ages.

In this direction, we compare our estimates of compressive strength at late ages with experimental data [71, 72, 17] provided as a function of the gel-space ratio. The gel-space ratio $\gamma$ is defined as the ratio between the volume of gel (i.e. hydration products plus "gel" micropores) and the sum of the volumes of gel and capillary pores [73. This parameter is often associated with the strength of cement-based materials. For instance, the compressive strength of the cement paste $f_{c, c p}$ is reported to scale with $\gamma^{n}$, with $n$ between 2 and 3 74. As can be seen in Figure 17 (right), our estimates at late ages are in agreement with other results regarding the relation of the compressive strength of cement pastes with gel-space ratio.

\subsection{Fluctuations in the presence of transformation fields}

In this section, we compute the fluctuations of stress fields accounting for the presence of transformation fields. Thermoelastic and poroelastic scenarios 

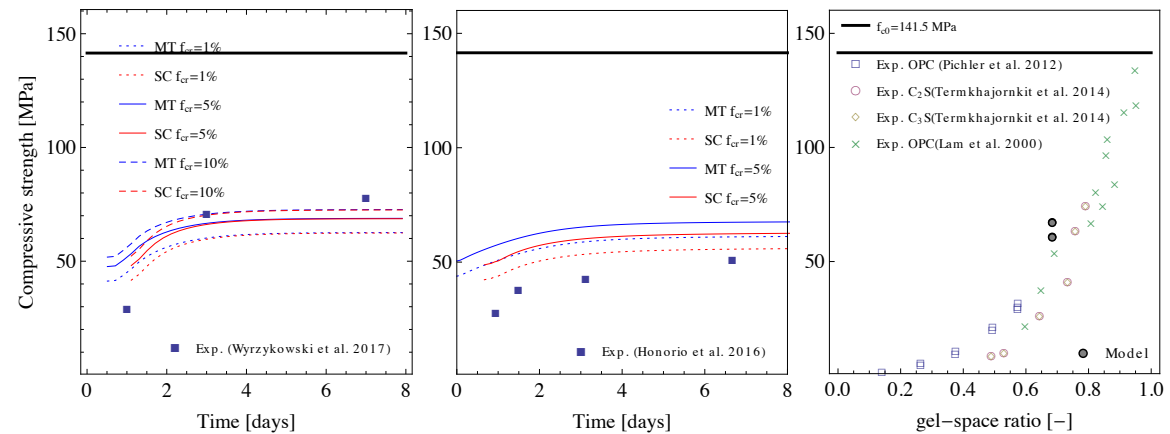

Figure 17: Comparison of analytical estimates with experimental compressive strength of cement pastes obtained from the literature 75, 4, 71, 72, 17. At left, the results of Wyrzykowski et al. 4 refer to a w/c of 0.3; at the center the results of Honorio et al. 75] refer to a w/c of 0.50. In both cases, the evolution of Young moduli with age are presented in the respective articles. Analytical estimations are provided as a function of the critical volume fraction $f_{c r}$ and homogenization scheme (MT ou SC) used to get the effective properties of the hydration product layer scale. 
are considered. As can be seen in Eq. 32 of the Appendix, the fluctuations are a sum of a "mechanical" load contribution, a transformation field contribution and a contribution of a coupling term.

Figure 18 shows the standard deviation of stresses at concrete scale according to a thermal loading corresponding to an increment of $T$ Kelvins in MT scenario described in the last section. The coefficients of thermal expansion of the mortar and aggregate are, respectively, $1 \times 10^{-5} \mathrm{~K}^{-1}$ and $0.8 \times 10^{-5} \mathrm{~K}^{-1}$ (e.g. [10]): there is, therefore, a mismatch between the transformation fields applied in each phase. The evolution of mortar bulk modulus is considered. We assume that the coefficient of thermal expansion of the mortar remains constant at early-age since this property is reported to vary only at the very early-ages. The results are shown in the absence of macroscopic mechanical loading $\left(\sigma_{0}=0\right)$ as well as for some values of macroscopic loading with an order of magnitude similar to that produced by the thermal load. A thermal load corresponding to an increment of $1 \mathrm{~K}$ induces standard deviation of stresses in the order of magnitude of $10 \mathrm{kPa}$. Again, the fluctuations, now due to a thermal load, are larger in the stiffer phase (the aggregate) and decrease with the age. The fluctuations due to thermoelastic transformation field and mechanical loads seem nearly independent for the system studied (i.e. the contribution of the coupling term is not significant).

Similarly, to evaluate the fluctuations due to a poromechanical load, a pore pressure of $1 \mathrm{MPa}$ applied in the capillary pores assumed saturated can be considered. The analysis is simplified by considering that this pressure is only applied in the mortar phase in a homogeneous way. The effective pressure applied in the mortar is corrected with respect to the capillary porosity (computed with hydration modelling presented in Section 4.3.1), being thus time-dependent. In this scenario, the standard deviation induced by a $1 \mathrm{MPa}$ of pore pressure is in the order of magnitude of 0.1 MPa. Physico-chemical processes may induce pore pressures of this order of magnitude. According to Flatt et al. [76], pressures in the order of $1 \mathrm{MPa}$ may take place in porous media due to salt crystallization. The resulting fluctuations may contribute to the onset of damage of CBM, 


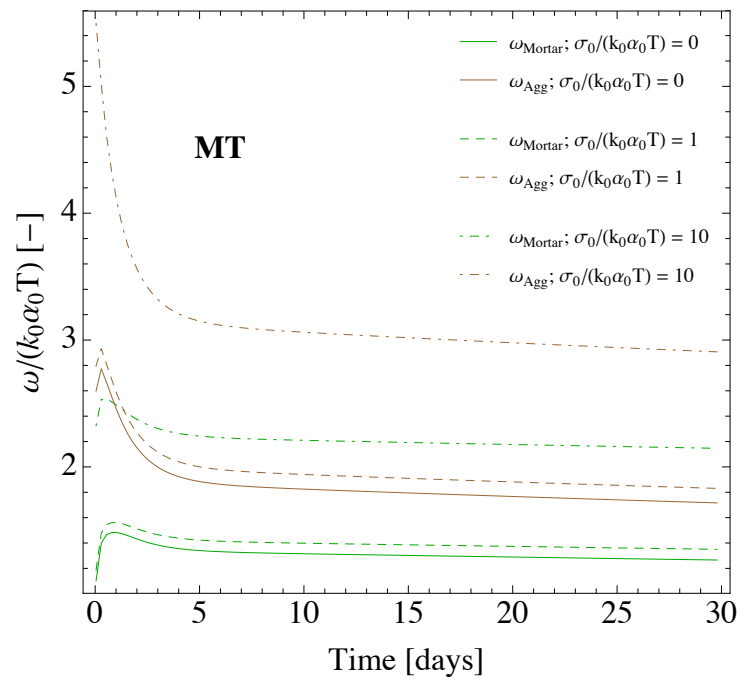

Figure 18: Standard deviation of stresses at concrete scale according to a thermal loading corresponding to an increment of $T$ Kelvins in MT scenario $\left(k_{0}=1 \mathrm{GPa}, \alpha_{0}=10^{-5} \mathrm{~K}^{-1}\right)$. 
which presents tensile strengths of few MPa.

\section{Conclusion}

In this paper, we propose, for the first time to the author's knowledge, estimations of the statistical variability of mechanical fields in cement-based materials at early-age by means of analytical homogenization. The estimations of fluctuations provided here are caused by the mismatch of mechanical properties of constituent phases in a cement-based composite. The phases were represented by isotropic spherical particles. Non-spherical and anisotropic particles are expected to experience larger fluctuations of mechanical fields. Therefore, our estimations can be viewed as a "minimal" value of fluctuation of mechanical fields in composites due to the mismatch of mechanical properties, which other fluctuations due to morphology and anisotropy can be added to. Since stress fields measurements in cement-based materials seem not to be available experimentally to date, theoretical modelling as the one proposed here arises as a potential tool to fill this gap. Note that at molecular scale, more fundamental fluctuations of mechanical fields can be observed due to atoms vibration within a single phase [77].

A direct way of using the estimations of the fluctuations of stresses is in the computation of the strength of composites. In our estimations of compressive strength of cement pastes at various $\mathrm{w} / \mathrm{c}$ ratios, under the assumption of spherical (or equiaxed) inclusions and micro- and macro-isotropy, we obtained reasonable agreement with experimental data, especially at late ages. This indirectly corroborates the suitability of the theory employed here to estimate fluctuations of mechanical fields in cement-based materials. In the sense of Popper, our results could be falsified if estimations of strength using the same theory are proven wrong. Furthermore, our results indicate that the choice of homogenization scheme and assumptions regarding anisotropy and microstructure morphology impacts decisively the estimations of strength of composites, as expected. 
Fluctuations increase with the stiffness of the phases. Stiffer aggregates display larger fluctuations in stresses (as well as larger average stresses per se). Also, sand and clinker particles are the most loaded phases and present the larger standard deviation of stresses in mortar and cement paste scales, respectively. The same occurs at the scale of hydration products with aFm phase. ITZ, being the less stiffer phase at mortar scale, exhibits lower average stresses and fluctuations at this scale. The fluctuations also increase with the contrast between the stiffness of constituents. Concretes with aggregates with similar stiffness than mortar exhibit less fluctuations of stresses in both phases compared to concretes with higher contrast.

Regarding the dependence of homogenization scheme in the estimation of fluctuations, the application to C-S-H shows that MT and SC schemes yield similar results for low porosities (below circa $35 \%$ ). The estimations of the standard deviation of stresses computed with both schemes in HD and LH CS-H have the order of magnitude of half the load applied on the bulk material. Two effects are observed in a multiscale framework: 1) localization of stresses in stiffer phases to the detriment of less stiff phases; 2) propagation of fluctuations towards smaller scales as detailed in Section 3.2. These effects are competing in the determination of the statistical variability of stresses within hydration products. For C-S-H, standard deviation of stresses for a hydrostatic load are of the order of magnitude of the stresses applied at concrete scale even if C-S-H is much less stiffer than other phases (AFm, aggregates, clinker) present across the scales.

Concerning the effect of age, different behaviours can be observed according to the scale considered. Discounted the very early-age (before 1 day), the standard deviation of stresses decreases with age at concrete and mortar scales and increases in hydration products scales. In the latter case, the solidification of the material can be used to explain the evolution. In the former case, the diminution of the contrast of stiffness may be evoked.

Finally, even in the absence of purely mechanical load, transformation fields such as thermoelastic and poroelastic loadings can induce fluctuations in me- 
chanical fields within composites. In the presence of mechanical load, both contributions to the fluctuations are added up together with a coupled contribution. In the example considered here, at concrete scale, a thermal load corresponding to an increment of $1 \mathrm{~K}$ induced a standard deviation of stresses in the order of magnitude of $10 \mathrm{kPa}$. Also, the standard deviation induced solely by a $1 \mathrm{MPa}$ of pore pressure was in the order of magnitude of $0.1 \mathrm{MPa}$.

\section{Acknowledgement}

The financial support of the French National Research Agency (ANR) through the project TEAM2ClayDesicc (ANR-14-CE05-0023-01) is gratefully acknowledged.

\section{Appendix A. Fluctuations for macro-isotropic composites in the pres- ence of transformation fields}

The evaluation of field fluctuations for macro-isotropic materials with spherical Eshelby inclusions is briefly presented based on the works of [24, 25] in thermoelasticity. However, we reason here in terms of general transformations fields, instead of eigenstrains due to uniform temperature change as in [25]. In their derivation, the strain fluctuations present three contributions: 1) $q_{p}^{\sigma, m}$, a contribution independent of the transformation field, 2) $q_{p}^{\sigma, t}$, a contribution depending only on the transformation field and 3) $q_{p}^{\sigma, m t}$, a coupled contribution:

$$
q_{p}^{\sigma}=q_{p}^{\sigma, m}+q_{p}^{\sigma, m t}+q_{p}^{\sigma, t}
$$

These three contributions can be computed from the same expression, based on the fluctuations of strain fields $q_{p}^{\varepsilon, X}$, by means of some transformations [25]:

$$
q_{p}^{\sigma, X} \equiv\langle\sigma \otimes \sigma\rangle_{p}-\langle\sigma\rangle_{p} \otimes\langle\sigma\rangle_{p}=C_{p} q_{p}^{\varepsilon, X}=C_{p} \mathbb{F}_{p}\langle\sigma\rangle \otimes\langle\sigma\rangle
$$

where the superscript $X=m, t$ or $m t$ according to the contributions mentioned above and the forth-rank tensor $\mathbb{F}^{p}$, whose components are defined in the following. 
In [25], the forth-rank tensor $q_{p}^{\varepsilon, m}$ is built in terms of second-rank tensors $I$ and $\bar{\varepsilon}$ where the strain $\bar{\varepsilon}=\mathbb{S}: \sigma$ denotes the mechanical contribution in the absence of a transformation field and $\bar{e}=\mathbb{K}: \bar{\varepsilon}=\bar{\varepsilon}-\operatorname{Tr} \bar{\varepsilon} / 3 \mathrm{I}$ is its deviatoric part. In this sense, the following forth-rank tensors are defined:

$$
\begin{aligned}
E_{0} & =I \otimes I \\
E_{1} & =\bar{e} \otimes I+I \otimes \bar{e} \\
E_{2} & =(\bar{\varepsilon}: \bar{\varepsilon}) \otimes I+I \otimes(\bar{e}: \bar{e}) \\
E_{3} & =\bar{e} \otimes \bar{e} \\
\left(\widetilde{E}_{n}\right)_{i k l m} & =\left(E_{n}\right)_{i k l m}+\left(E_{n}\right)_{i l k m}+\left(E_{n}\right)_{i m l k}
\end{aligned}
$$

where the tensors $\widetilde{E}_{n}$ are the symmetrized versions of the $E_{n}$ tensors $(n=$ $0,1,2,3)$.

Thus, the first contribution reads:

$$
\begin{aligned}
q_{p}^{\varepsilon, m}= & {\left[F_{11}(\operatorname{Tr} \bar{\varepsilon} / 3)^{2}+\left(F_{12} / \sqrt{5}\right) \operatorname{Tr} \bar{e}^{2} / 3\right] E_{0} } \\
& +\left[\left(F_{21} / 2 \sqrt{5}\right)(\operatorname{Tr} \bar{\varepsilon} / 3)^{2}+\left(F_{22} / 10\right) \operatorname{Tr} \bar{e}^{2} / 3\right]\left(3 \widetilde{E}_{0}-5 E_{0}\right) \\
& +\left(F_{33} / 21\right)\left[2 \operatorname{Tr} \bar{e}^{2} \widetilde{E}_{0} 5+7 \widetilde{E}_{3}-2 \widetilde{E}_{2}\right] \\
& +F_{44}(\operatorname{Tr} \bar{\varepsilon} / 3)^{2} E_{1}+\left(4 F_{45} / \sqrt{14}\right)\left[\left(\operatorname{Tr} \bar{e}^{2} / 3\right) E_{0}-E_{2} / 2\right] \\
& -\left(F_{54} / \sqrt{14}\right)(\operatorname{Tr} \bar{\varepsilon} / 3)\left(3 \widetilde{E}_{1}-7 E_{1}\right) \\
& -\left(F_{55} / 7\right)\left[2\left(\operatorname{Tr} \bar{e}^{2} / 3\right)\left(3 \widetilde{E}_{0}-7 E_{0}\right)-\left(3 \widetilde{E}_{2}-7 E_{2}\right)\right]
\end{aligned}
$$

where the $p$ superscript of the components of the tensor $\mathbb{F}^{p}$ was omitted for conciseness; $\operatorname{Tr}$ is the trace operator.

The other contributions can be obtained from Eq. 35 by the substitutions [25], $(\operatorname{Tr} \bar{\varepsilon} / 3)^{2} \rightarrow 2 \zeta_{1}(\operatorname{Tr} \bar{\varepsilon} / 3) \theta$ and $\operatorname{Tr} \bar{\varepsilon} / 3 \rightarrow \zeta_{1}^{\prime} \theta$ for $q_{p}^{\varepsilon, m t}$; and $(\operatorname{Tr} \bar{\varepsilon} / 3)^{2} \rightarrow$ $\zeta_{2} \theta^{2}$ for $q_{p}^{\varepsilon, t}$ :

$$
\begin{aligned}
q_{p}^{\varepsilon, m t}= & {\left[2 F_{11} \zeta_{1}(\operatorname{Tr} \bar{\varepsilon} / 3) E_{0}\right.} \\
& +\left(F_{21} \zeta_{1} / \sqrt{5}\right)(\operatorname{Tr} \bar{\varepsilon} / 3)\left(3 \widetilde{E}_{0}-5 E_{0}\right) \\
& \left.+F_{44} \zeta_{1}^{\prime} E_{1}-\left(F_{54} \zeta_{1}^{\prime} / \sqrt{14}\right)\left(3 \widetilde{E}_{1}-7 E_{1}\right)\right]
\end{aligned}
$$




$$
q_{p}^{\varepsilon, t}=\left[F_{11} E_{0}+\left(F_{21} / 2 \sqrt{5}\right)\left(3 \widetilde{E}_{0}-5 E_{0}\right)\right] \zeta_{2}
$$

The components of tensor $\mathbb{F}^{p}$ are given in terms of the eighth-rank tensor $\mathcal{H}$ that in the isotropic case owns 10 independent components:

$$
\begin{array}{ll}
F_{11}=f_{p}^{2} H_{12} H_{21} / D_{12} & F_{11}=f_{p} g_{p}\left(1-H_{55}-D_{45}\right) / D_{45} \\
F_{12}=f_{p}^{2} H_{12} / D_{12} & F_{45}=f_{p} g_{p} H_{45} / D_{45} \\
F_{21}=g_{p}^{2} H_{21} / D_{12} & F_{54}=g_{p}^{2} H_{54} / D_{45} \\
F_{22}=g_{p}^{2}\left(1-D_{12}\right) / D_{12} & F_{55}=g_{p}^{2}\left(1-H_{44}-D_{45}\right) / D_{45} \\
F_{33}=g_{p}^{2} H_{33} /\left(1-H_{33}\right) &
\end{array}
$$

where

$$
\begin{array}{ll}
D_{12}=1-H_{22}-H_{12} H_{21} & D_{45}=\left(1-H_{44}\right)\left(1-H_{55}\right)-H_{45} H_{54} \\
H_{11}=0 & H_{45}=-10 \sqrt{\frac{2}{7}}(5-7 v)(1-2 v) h_{1} \\
H_{12}=50 \sqrt{1 / 5}(1-2 v)^{2} h_{1} & H_{54}=50 \sqrt{1 / 5}(5-7 v) h_{3} \\
H_{21}=\sqrt{4 / 5} h_{2} & H_{55}=-\frac{3}{7}\left(11-50 \nu+35 \nu^{2}\right) h_{1} \\
H_{22}=2\left(23-50 \nu+35 \nu^{2}\right) h_{1} & H_{33}=H_{66}=\frac{4}{7}\left(18-50 \nu+35 \nu^{2}\right) h_{1} \\
H_{44}=2(1-2 v) h_{3} &
\end{array}
$$

and

$$
\begin{gathered}
f_{p}=1-3 A_{p}^{j} \quad g_{p}=1-2 A_{p}^{k} \\
h_{1}=\frac{\left\langle\left(A_{p}^{k}\right)^{2}\right\rangle_{\Omega}}{(4-5 v)^{2}} \quad h_{2}=\left\langle\left(3 A_{p}^{k}\right)^{2}\right\rangle_{\Omega} \quad h_{3}=\frac{\left\langle 3 A_{p}^{k} A_{p}^{j}\right\rangle_{\Omega}}{4-5 v}
\end{gathered}
$$

where the hydrostatic $A_{p}^{j}$ and deviatoric $A_{p}^{k}$ components of strain localization tensor, respectively. The last term depend on the effective Poisson ratio of the 765 composite $(\nu=(3 k-2 \mu) /[2(3 k+\mu)])$. 
The coefficients $\zeta$ in Eqs. 35 and 36 are:

$$
\zeta_{1}=\frac{\left\langle\left(A_{p}^{j}\right)^{2}\langle\omega\rangle_{p}\right\rangle_{\Omega}}{\left\langle\left(A_{p}^{j}\right)^{2}\right\rangle_{\Omega}} \quad \zeta_{1}^{\prime}=\frac{\left\langle A_{p}^{j} A_{p}^{k}\langle\omega\rangle_{p}\right\rangle_{\Omega}}{\left\langle A_{p}^{j} A_{p}^{k}\right\rangle_{\Omega}} \quad \zeta_{2}=\frac{\left\langle A_{p}^{k}\left(\langle\omega\rangle_{p}\right)^{2}\right\rangle_{\Omega}}{\left\langle\left(A_{p}^{k}\right)^{2}\right\rangle_{\Omega}}
$$

From Eq. 9, in the micro-isotropic case and considering a general transformation field

$$
\langle\omega\rangle_{p}=\frac{k_{p}}{\left(k_{p}-k\right)}\left(\varepsilon^{(0)}-\varepsilon_{p}^{(0)}\right)
$$

For comparison, in the case of uniform temperature change $\theta$, we have as

in 25. (Eq. 2.29), $\left\langle\sigma^{r e s}\right\rangle_{p}=\omega_{p} \theta=\frac{k_{p}}{\left(k_{p}-k\right)}\left(\alpha-\alpha_{p}\right) \theta$, where $\alpha$ and $\alpha_{p}$ are, respectively, the effective and per phase coefficients of thermal expansion.

Finally, to get the stress fluctuations from strains fluctuations as presented in Eq. 33, the transformations $F_{i k} \rightarrow C_{p}^{i} F_{i k}$ and $\bar{\varepsilon} \rightarrow \bar{\sigma}$ are performed in Eqs. 35, 36 and 37, with

$$
\begin{gathered}
C_{p}^{1}=\left(3 k_{p}\right)^{2} \quad C_{p}^{2}=6 k_{p} \mu_{p} \quad C_{p}^{3}=\left(2 \mu_{p}\right)^{2} \\
\operatorname{Tr} \bar{\varepsilon} \rightarrow \operatorname{Tr} \bar{\sigma} / 3 k \quad \bar{e} \rightarrow \bar{s}=\mathbb{K}: \bar{\sigma}=(\bar{\sigma}-\operatorname{Tr} \bar{\sigma} / 3 \mathrm{I}) / 2 \mu
\end{gathered}
$$

[ [1] O. Bernard, F.-J. Ulm, E. Lemarchand, A multiscale micromechanicsa hydration model for the early-age elastic properties of cement-based materials, Cement and Concrete Research 33 (9) (2003) 1293-1309.

URL http://www.sciencedirect.com/science/article/pii/ S0008884603000395

q [2] J. Sanahuja, L. Dormieux, G. Chanvillard, Modelling elasticity of a hydrating cement paste, Cement and Concrete Research 37 (10) (2007) 1427-1439.

口 URL http://www.sciencedirect.com/science/article/pii/ S0008884607001548 
[3] T. Honorio, B. Bary, F. Benboudjema, Estimation of Elastic Properties of Cement based Materials at Early Age based on a Combined Numerical and Analytical Multiscale Micromechanics Approach, in: RILEM International Symposium on Concrete Modelling, Beijing, China, 2014.

[4] M. Wyrzykowski, J. Sanahuja, L. Charpin, M. Knigsberger, C. Hellmich, B. Pichler, L. Valentini, T. Honrio, V. Smilauer, K. Hajkova, G. Ye, P. Gao, C. Dunant, A. Hilaire, S. Bishnoi, M. Azenha, Numerical benchmark campaign of COST Action TU1404 microstructural modelling, RILEM Technical Letters 2 (2017) 99-107. doi:10.21809/rilemtechlett.2017.44. URL https://letters.rilem.net/index.php/rilem/article/view/44

[5] J. Sanahuja, Homogenization of Solidifying Random Porous Media: Application to Ageing Creep of Cementitious Materials, Beijing, China, 2014.

口[6] T. Honorio, B. Bary, F. Benboudjema, Multiscale estimation of a ageing viscoelastic properties of cement-based materials: A com${ }_{800}$ a bined analytical and numerical approach to estimate the behaviour at early age, Cement and Concrete Research 85 (2016) 137-155. doi:10.1016/j.cemconres.2016.03.010 URL http://www.sciencedirect.com/science/article/pii/ S0008884615301411

805 [7] S. Scheiner, C. Hellmich, Continuum microviscoelasticity model for aging basic creep of early-age concrete, Journal of engineering mechanics 135 (4) (2009) 307-323. URL http://ascelibrary.org/doi/abs/10.1061/(ASCE) 0733-9399(2009)135:4(307)

${ }_{810}^{\text {口 }}[8]$ A. Aili, M. Vandamme, J.-M. Torrenti, B. Masson, Theoretical and a practical differences between creep and relaxation Poissons ratios in linear viscoelasticity, Mechanics of Time-Dependent Materials (2015) 1-19doi:10.1007/s11043-015-9277-5. 
URL

http://link.springer.com/article/10.1007/

[9] B. Bary, S. Bjaoui, Assessment of diffusive and mechanical proper-

1 ties of hardened cement pastes using a multi-coated sphere assemblage model, Cement and Concrete Research 36 (2) (2006) 245-258. doi:10.1016/j.cemconres.2005.07.007.

URL http://linkinghub.elsevier.com/retrieve/pii/ S0008884605001973

[10] T. Honorio, B. Bary, F. Benboudjema, Thermal properties of cement-based materials: Multiscale estimations at early-age, Cement and Concrete Composites 87 (2018) 205-219. doi:10.1016/j.cemconcomp.2018.01.003

[11] D. P. Bentz, Transient plane source measurements of the thermal properties of hydrating cement pastes, Materials and Structures 40 (10) (2007) 1073-1080. doi:10.1617/s11527-006-9206-9.

URL http://link.springer.com/article/10.1617/ s11527-006-9206-9

[12] S. Ghabezloo, Micromechanics analysis of thermal expansion and thermal pressurization of a hardened cement paste, Cement and Concrete Research 41 (5) (2011) 520-532. doi:10.1016/j.cemconres.2011.01.023. URL https://hal.archives-ouvertes.fr/hal-00561857

[13] L. Ponson, Statistical aspects in crack growth phenomena: how the fluctuations reveal the failure mechanisms, International Journal of Fracture (2016) 1-17doi:10.1007/s10704-016-0117-7.

(1) URL http://link.springer.com/article/10.1007/

840 s10704-016-0117-7

[14] L. Dormieux, A. Molinari, D. Kondo, Micromechanical approach to the behavior of poroelastic materials, Journal of the Mechanics and Physics of 
Solids 50 (10) (2002) 2203-2231. doi:10.1016/S0022-5096(02) 00008-X.

URL http://www.sciencedirect.com/science/article/pii/

[15] B. Pichler, C. Hellmich, Upscaling quasi-brittle strength of cement paste and mortar: A multi-scale engineering mechanics model, Cement and Concrete Research 41 (5) (2011) 467-476. doi:10.1016/j.cemconres.2011.01.010. S0008884611000111

[16] M. Knigsberger, B. Pichler, C. Hellmich, Micromechanics of ITZAggregate Interaction in Concrete Part I: Stress Concentration, Journal of the American Ceramic Society 97 (2) (2014) 535-542. doi:10.1111/jace.12591.

URL http://onlinelibrary.wiley.com/doi/10.1111/jace.12591/ abstract

[17] B. Pichler, C. Hellmich, J. Eberhardsteiner, J. Wasserbauer, P. Termkhaa jornkit, R. Barbarulo, G. Chanvillard, Effect of gelspace ratio and

a microstructure on strength of hydrating cementitious materials: An 860 engineering micromechanics approach, Cement and Concrete Research 45 (2013) 55-68. doi:10.1016/j.cemconres.2012.10.019.

a URL http://linkinghub.elsevier.com/retrieve/pii/ S0008884612002384

[18] F. Lavergne, A. Ben Fraj, I. Bayane, J. F. Barthlmy, Estimating the 865 mechanical properties of hydrating blended cementitious materials: An investigation based on micromechanics, Cement and Concrete Researchdoi:10.1016/j.cemconres .2017.10.018.

1. URL https://www.sciencedirect.com/science/article/pii/ S0008884617304015

870 [19] A. Sellier, S. Multon, L. Buffo-Lacarrire, T. Vidal, X. Bourbon, a G. Camps, Concrete creep modelling for structural applications: non- 
linearity, multi-axiality, hydration, temperature and drying effects,

Cement and Concrete Research 79 (Supplement C) (2016) 301-315.

doi:10.1016/j.cemconres.2015.10.001.

875

URL http://www.sciencedirect.com/science/article/pii/

S0008884615002616

[20] M. Bauchy, M. J. A. Qomi, C. Bichara, F.-J. Ulm, R. J.-M. Pellenq, Topo-

a logical Origin of Fracture Toughening in Complex Solids: the Viewpoint of Rigidity Theory, arXiv:1410.2916 [cond-mat]ArXiv: 1410.2916. URL http: //arxiv .org/abs/1410.2916

[21] Z. P. Bazant, A. B. Hauggaard, S. Baweja, F.-J. Ulm, Microprestresssolidification theory for concrete creep. I: Aging and drying effects, Journal of Engineering Mechanics 123 (11) (1997) 1188-1194.

1. URL http://ascelibrary.org/doi/abs/10.1061/(ASCE) 0733-9399(1997)123\%3A11(1188)

[22] P. Rossi, J.-L. Tailhan, F. Le Maou, L. Gaillet, E. Martin, Basic creep behavior of concretes investigation of the physical mechanisms by using acoustic emission, Cement and concrete research 42 (1) (2012) 61-73.

[23] W. Kreher, W. Pompe, Field fluctuations in a heterogeneous elastic materialan information theory approach, Journal of the Mechanics and Physics of Solids 33 (5) (1985) 419-445. doi:10.1016/0022-5096(85)90008-0.

口 URL http://www.sciencedirect.com/science/article/pii/ 0022509685900080

[24] M. Bobeth, G. Diener, Field fluctuations in multicomponent mixtures, Journal of the Mechanics and Physics of Solids 34 (1) (1986) 1-17. doi:10.1016/0022-5096(86)90002-5. URL http://www.sciencedirect.com/science/article/pii/ 0022509686900025

[25] M. Bobeth, G. Diener, Static elastic and thermoelastic field fluctuations in multiphase composites, Journal of the Mechanics and Physics of Solids 
35 (2) (1987) 137-149. doi:10.1016/0022-5096(87)90033-0.

URL http://www.sciencedirect.com/science/article/pii/ 0022509687900330

[26] V. A. Buryachenko, F. G. Rammerstorfer, Thermoelastic stress fluctuations in random-structure coated particulate composites, European Journal of Mechanics - A/Solids 17 (5) (1998) 763-788. doi:10.1016/S0997-7538(98)80004-1. URL http://www.sciencedirect.com/science/article/pii/ S0997753898800041

910

[30] T. Honorio, B. Bary, F. Benboudjema, Evaluation of the contribution massive concrete structure, Engineering Structures 80 (2014) 173-188. doi:10.1016/j.engstruct.2014.08.050.

925 URL http://ww.sciencedirect.com/science/article/pii/ S0141029614005215

[31] T. Honorio, L. Brochard, Multiscale Estimation of the Fluctuations of Me口 chanical Fields in Poroelasticity: Application to Cement-Based Compos- 
ites, Poromechanics Vldoi:10.1061/9780784480779.164.

[32] G. J. Dvorak, Y. Benveniste, On Transformation Strains and Uniform Fields in Multiphase Elastic Media, Proceedings of the Royal Society of London A: Mathematical, Physical and Engineering Sciences 437 (1900) (1992) 291-310. doi:10.1098/rspa.1992.0062.

935 U URL http://rspa.royalsocietypublishing.org/content/437/1900/ 291

[33] J. Eshelby, Progress in solid mechanics: Vol. 1. Edited by IN Sneddon and R. Hill, North-Holland Publishing Company, Amsterdam, 1960, 448 pp., 100s.

${ }_{940}$ [34] T. Mura, Micromechanics of defects in solids, 2nd Edition, Mechanics of elastic and inelastic solids. Editors: S. Nemat-Nasser and G. AE. Oravas, Martinus Nijhoff Publishers, 1987.

[35] R. Hill, Continuum micro-mechanics of elastoplastic polycrystals, Journal of the Mechanics and Physics of Solids 13 (2) (1965) 89-101. doi:10.1016/0022-5096(65)90023-2.

I URL http://www.sciencedirect.com/science/article/pii/ 0022509665900232

[36] N. Laws, On the thermostatics of composite materials Journal of the Mechanics and Physics of Solids 21 (1) (1973) 9-17. doi:10.1016/0022-5096(73)90027-6. URL http://www.sciencedirect.com/science/article/pii/ 0022509673900276

[37] M. Bornert, R. Masson, P. P. Castaeda, A. Zaoui, Second-order estimates for the effective behaviour of viscoplastic polycrystalline materials, Journal of the Mechanics and Physics of Solids 49 (11) (2001) 2737-2764. doi:10.1016/S0022-5096(01)00077-1. 
URL http://www.sciencedirect.com/science/article/pii/ S0022509601000771

[38] G. Siboni, Y. Benveniste, A micromechanics model for the effective

[39] V. M. Levin, Thermal Expansion Coefficients of Heterogeneous Materials,

[40] E. Herve, A. Zaoui, n-Layered inclusion-based micromechanical modelling, International Journal of Engineering Science 31 (1) (1993) 1-10. doi:10.1016/0020-7225(93)90059-4.

口

970

[41] Z. Hashin, Thermoelastic properties of particulate composites with imperfect interface, Journal of the Mechanics and Physics of Solids 39 (6) (1991) 745-762. doi:10.1016/0022-5096(91)90023-H.

1 URL http://www.sciencedirect.com/science/article/pii/ $975 \quad 002250969190023 \mathrm{H}$

[42] Z. Hashin, P. J. M. Monteiro, An inverse method to determine the elastic properties of the interphase between the aggregate and the cement paste Cement and Concrete Research 32 (8) (2002) 1291-1300. URL http://www.sciencedirect.com/science/article/pii/ S0008884602007925

[43] R. Brenner, O. Castelnau, P. Gilormini, A modified affine theory for the overall properties of nonlinear composites, Comptes Rendus de l'Acadmie des Sciences - Series IIB - Mechanics 329 (9) (2001) 649-654. doi:10.1016/S1620-7742(01)01382-4. 
URL http://www.sciencedirect.com/science/article/pii/ S1620774201013824

[44] R. Brenner, O. Castelnau, L. Badea, Mechanical field fluctuations in polycrystals estimated by homogenization techniques, Proceedings of the Royal Society of London A: Mathematical, Physical and Engineering Sciences 460 (2052) (2004) 3589-3612. doi:10.1098/rspa.2004.1278. URL http://rspa.royalsocietypublishing.org/content/460/2052/ 3589

[45] V. A. Buryachenko, W. S. Kreher, Internal residual stresses in heterogeneous solidsA statistical theory for particulate composites, Journal of the Mechanics and Physics of Solids 43 (7) (1995) 1105-1125. doi:10.1016/0022-5096(95)00029-I. URL http://www.sciencedirect.com/science/article/pii/ $002250969500029 \mathrm{I}$

[46] B. Bary, C. Bourcier, T. Helfer, Analytical and 3d numerical analysis 1000 of the thermoviscoelastic behavior of concrete-like materials including interfaces, Advances in Engineering Software 112 (2017) 16-30. doi:10.1016/j.advengsoft.2017.06.006

1. URL http://www.sciencedirect.com/science/article/pii/ S0965997816305452

1005 [47] T. de Larrard, B. Bary, E. Adam, F. Kloss, Influence of aggregate a shapes on drying and carbonation phenomena in 3d concrete numerical samples, Computational Materials Science 72 (2013) 1-14. doi:10.1016/j.commatsci.2013.01.039.

1 URL http://www.sciencedirect.com/science/article/pii/ S0927025613000566

[48] T. Nguyen, B. Bary, T. De Larrard, Coupled carbonation-rust formationa damage modeling and simulation of steel corrosion in $3 \mathrm{~d}$ mesoscale reinforced concrete, Cement and Concrete Research 74 (2015) 95-107. 


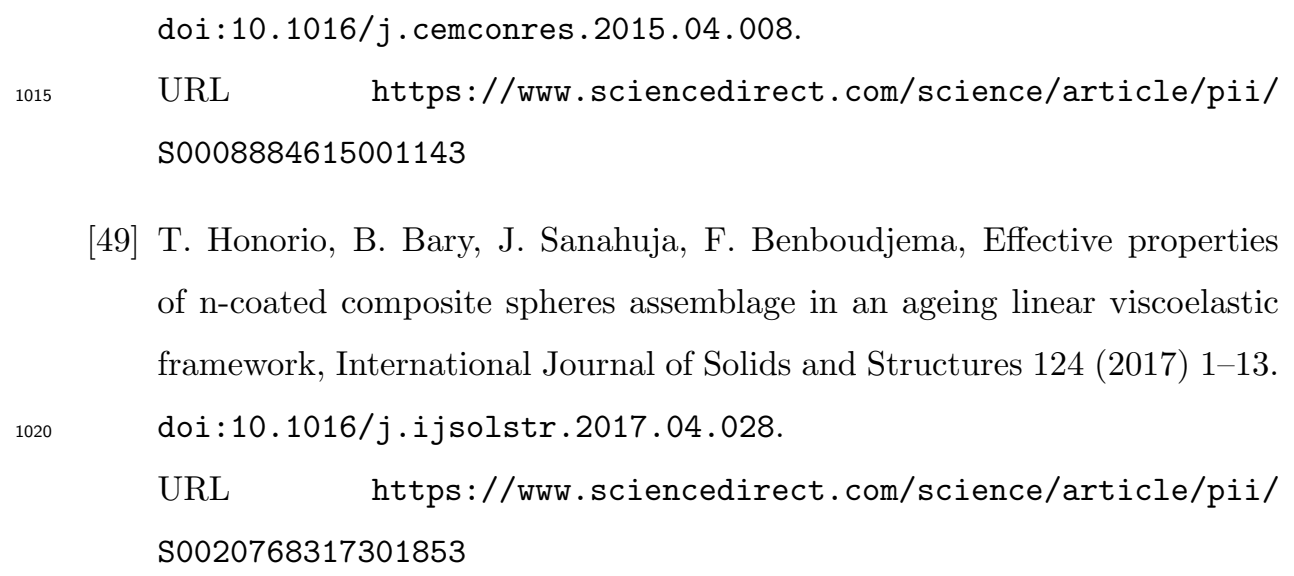

[49] T. Honorio, B. Bary, J. Sanahuja, F. Benboudjema, Effective properties

a of $\mathrm{n}$-coated composite spheres assemblage in an ageing linear viscoelastic framework, International Journal of Solids and Structures 124 (2017) 1-13.

[50] F. Bernachy-Barbe, B. Bary, Effect of numerical versus tomographic aggregate shapes in a $3 \mathrm{~d}$ mesoscale approach to concrete creep, (submitted paper).

[51] G. Constantinides, F.-J. Ulm, The effect of two types of C-S-H on the elasticity of cement-based materials: Results from nanoindentation and micromechanical modeling, Cement and Concrete Research 34 (1) (2004) 67-80. doi:10.1016/S0008-8846(03)00230-8. URL http://www.sciencedirect.com/science/article/pii/ S0008884603002308

[52] K. Ioannidou, K. J. Krakowiak, M. Bauchy, C. G. Hoover, E. Masoero, 口 S. Yip, F.-J. Ulm, P. Levitz, R. J.-M. Pellenq, E. D. Gado, Mesoscale texture of cement hydrates, Proceedings of the National Academy of Sciences (2016) 201520487doi:10.1073/pnas.1520487113. URL http://www .pnas.org/content/early/2016/02/04/1520487113

[53] H. Laubie, S. Monfared, F. Radja, R. Pellenq, F.-J. Ulm, Disorderinduced stiffness degradation of highly disordered porous materials, Journal of the Mechanics and Physics of Solids 106 (2017) 207-228. doi:10.1016/j.jmps.2017.05.008. 
[54] M. Youssef, R. J.-M. Pellenq, B. Yildiz, Glassy Nature of Water in an Ultraconfining Disordered Material: The Case of CalciumSilicateHydrate, Journal of the American Chemical Society 133 (8) (2011) 2499-2510. doi: $10.1021 / \mathrm{ja107003a}$

URL http://dx.doi.org/10.1021/ja107003a

[55] P. Trtik, B. Mnch, P. Lura, A critical examination of statistical nanoinden-

a tation on model materials and hardened cement pastes based on virtual experiments, Cement and Concrete Composites 31 (10) (2009) 705-714. doi:10.1016/j.cemconcomp.2009.07.001

口 URL http://www.sciencedirect.com/science/article/pii/ S095894650900119X

[56] W. Wilson, L. Sorelli, A. Tagnit-Hamou, Automated coupling of 1055 NanoIndentation and Quantitative Energy-Dispersive Spectroscopy

(NI-QEDS): A comprehensive method to disclose the micro-chemomechanical properties of cement pastes, Cement and Concrete Researchdoi:10.1016/j.cemconres.2017.08.016. URL http://www.sciencedirect.com/science/article/pii/ S0008884617305549

[57] J. J. Chen, L. Sorelli, M. Vandamme, F.-J. Ulm, G. Chanvillard, A 1 Coupled Nanoindentation/SEMEDS Study on Low Water/Cement Ratio Portland Cement Paste: Evidence for $\mathrm{CSH} / \mathrm{Ca}(\mathrm{OH}) 2$ Nanocomposites, Journal of the American Ceramic Society 93 (5) (2010) 1484-1493. doi:10.1111/j.1551-2916.2009.03599.x.

a URL http://onlinelibrary.wiley.com/doi/10.1111/j.1551-2916. 2009.03599.x/abstract

[58] E. Stora, B. Bary, Q. C. He, E. Deville, P. Montarnal, Modn elling and simulations of the chemomechanical behaviour of leached 1070 cement-based materials: Leaching process and induced loss of stiffness, Cement and Concrete Research 39 (9) (2009) 763-772. 
doi:10.1016/j.cemconres.2009.05.010.

口

URL http://www.sciencedirect.com/science/article/pii/ S0008884609001240

[61] P. D. Tennis, H. M. Jennings, A model for two types of calcium silicate hydrate in the microstructure of Portland cement pastes, Cement and Concrete Research 30 (2000) 855-863.

[62] K. Velez, S. Maximilien, D. Damidot, G. Fantozzi, F. Sorrentino, De1090 termination by nanoindentation of elastic modulus and hardness of pure constituents of Portland cement clinker, Cement and Concrete Research 31 (4) (2001) 555-561.

口 URL http://www.sciencedirect.com/science/article/pii/ S0008884600005056

1095

[63] P. J. M. Monteiro, C. T. Chang, The elastic moduli of calcium hydroxide, Cement and Concrete Research 25 (8) (1995) 1605-1609. doi:10.1016/0008-8846(95) 00154-9.

a URL http://www.sciencedirect.com/science/article/pii/ 0008884695001549 
[64] S. Kamali, Comportement et simulation des matriaux cimentaires en environnement agressifs : Lixiviation et temprature, Ph.D. thesis, thse de doctorat dirige par Moranville, Micheline Sciences pratiques. Mcanique, gnie mcanique, gnie civil Cachan, Ecole normale suprieure 2003 2003DENS0004 (2003). URL http://www.theses.fr/2003DENS0004

[65] C.-J. Haecker, E. Garboczi, J. Bullard, R. Bohn, Z. Sun, S. Shah, T. Voigt, Modeling the linear elastic properties of Portland cement paste, Cement and Concrete Research 35 (10) (2005) 1948-1960. doi:10.1016/j.cemconres.2005.05.001. URL http://linkinghub.elsevier.com/retrieve/pii/ S0008884605001201

[66] E. Stora, Multi-scale modelling and simulations of the chemo-mechanical behavior of degraded cement-based materials, Ph.D. thesis, Universit ParisEst (2007).

[67] T. Honorio, Modelling concrete behaviour at early-age: multiscale analysis and simulation of a massive disposal structure, $\mathrm{PhD}$ Thesis, ENS Cachan (Univerist Paris-Saclay), France (2015).

[68] P. K. Mehta, J. M. Monteiro, Concrete: Microstructure, Properties, and Materials, 4th Edition, McGraw-Hill Professional, New York, 2013. mechanical properties of concrete, Construction and Building Materials 23 (8) (2009) 2821-2828. doi:10.1016/j.conbuildmat.2009.02.038.

1] URL http://www.sciencedirect.com/science/article/pii/ 1125

[70] B. Pichler, C. Hellmich, J. Eberhardsteiner, Spherical and acicular प representation of hydrates in a micromechanical model for cement paste: 
prediction of early-age elasticity and strength, Acta Mechanica 203 (3-4)

(2009) 137-162. doi:10.1007/s00707-008-0007-9.

s00707-008-0007-9

[71] P. Termkhajornkit, Q. H. Vu, R. Barbarulo, S. Daronnat, G. Chanvillard,

a Dependence of compressive strength on phase assemblage in cement

1 pastes: Beyond gelspace ratio Experimental evidence and microme-

1135 chanical modeling, Cement and Concrete Research 56 (2014) 1-11. doi:10.1016/j.cemconres.2013.10.007.

1) URL http://www.sciencedirect.com/science/article/pii/ S0008884613002160

[72] L. Lam, Y. L. Wong, C. S. Poon, Degree of hydration and gel/space ratio of high-volume fly ash/cement systems, Cement and Concrete Research 30 (5) (2000) 747-756. doi:10.1016/S0008-8846(00)00213-1. S0008884600002131

[73] T. C. Powers, T. L. Brownyard, Studies of the Physical Properties of Hardened Portland Cement Paste, Journal Proceedings 43 (9) (1946) 101-132. doi:10.14359/8745.

口 URL https://ww. concrete.org/publications/ internationalconcreteabstractsportal . aspx?m=details\&ID=8745

[74] D. P. Bentz, CEMHYD3d: A Three-Dimensional Cement Hydration and Microstructure Development Modeling Package. Version 3.0. NISTIR 7232. National Institute of Standards and Technology Interagency Report, Technology Administration, U.S. Department of Commerce, NISTIR 7232, Tech. rep. (Jun. 2005).

[75] T. Honorio, B. Bary, F. Benboudjema, Factors affecting the 1155 a thermo-chemo-mechanical behaviour of massive concrete structures at early-age, Materials and Structures 49 (8) (2016) 3055-3073. 


doi:10.1617/s11527-015-0704-5
URL https://link.springer.com/article/10.1617/
s11527-015-0704-5

[76] R. J. Flatt, F. Caruso, A. M. A. Sanchez, G. W. Scherer, Chemo-

mechanics of salt damage in stone, Nature Communications 5. doi: $10.1038 /$ ncomms5823

a URL http://www.nature.com/ncomms/2014/140911/ncomms5823/full/ ncomms5823.html

1165 [77] M. Parrinello, A. Rahman, Strain fluctuations and elastic constants, The घ Journal of Chemical Physics 76 (5) (1982) 2662-2666. doi:10.1063/1. 443248 .

URL http://aip.scitation.org/doi/abs/10.1063/1.443248 